\title{
Approximation of the two-fluid flow problem for viscoelastic fluids using the level set method and pressure enriched finite element shape functions
}

\author{
E. Castillo ${ }^{\mathrm{a}}$, J. Baiges ${ }^{\mathrm{a}, \mathrm{b}}$ and R. Codina $\mathrm{a}^{\mathrm{a}, \mathrm{b}}$ \\ ${ }^{a}$ Universitat Politècnica de Catalunya, Jordi Girona 1-3, Edifici C1, 08034, Barcelona, Spain \\ ${ }^{b}$ CIMNE - Centre Internacional de Metodes Numerics en Enginyeria, Gran Capità S/N 08034 Barcelona, Spain \\ ecastillo@cimne.upc.edu, jbaiges@cimne.upc.edu and ramon.codina@upc.edu
}

\begin{abstract}
The numerical simulation of complex flows has been a subject of intense research in the last years with important industrial applications in many fields. In this paper we present a finite element method to solve the two immiscible fluid flow problems using the level set method. When the interface between both fluids cuts an element, the discontinuity in the material properties leads to discontinuities in the gradients of the unknowns which cannot be captured using a standard finite element interpolation. The method presented in this work features a local enrichment for the pressure unknowns which allows one to capture pressure gradient discontinuities in fluids presenting different density values. The method is tested on two problems: the first example consists of a sloshing case that involves the interaction of a Giesekus and a Newtonian fluid. This example shows that the enriched pressure functions permit the exact resolution of the hydrostatic rest state. The second example is the classical jet buckling problem used to validate our method. To permit the use of equal interpolation between the variables, we use a variational multiscale formulation proposed recently by Castillo and Codina in Comput. Methods Appl. Mech. Engrg. 279 (2014) 579-605, that has shown very good stability properties, permitting also the resolution of the jet buckling flow problem in the the range of Weissenberg number $0<\mathrm{We}<100$, using the Oldroyd-B model without any sign of numerical instability. Additional features of the work are the inclusion of a discontinuity capturing technique for the constitutive equation and some comparisons between a monolithic resolution and a fractional step approach to solve the viscoelastic fluid flow problem from the point of view of computational requirements.

Keywords: Viscoelastic fluids, Level set method, Jet buckling, Oldroyd-B fluids, Giesekus fluids, Stabilized finite element methods.
\end{abstract}

\section{Contents}

1 Introduction $\quad 2$

2 The viscoelastic flow problem $\quad 4$

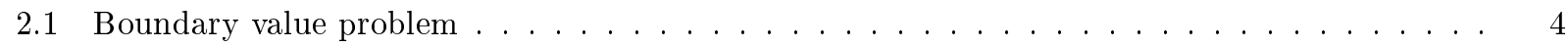

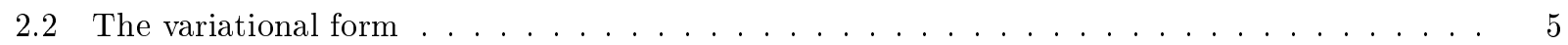

3 Numerical approximation $\quad 6$

3.1 Galerkin finite element discretization $\ldots \ldots \ldots \ldots \ldots$ 
3.2 Stabilized finite element formulation . . . . . . . . . . . . . . . . . 7

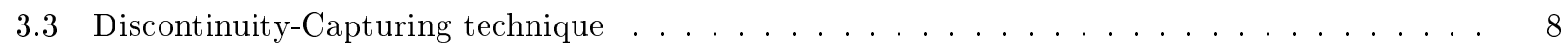

3.4 Monolithic time discretization . . . . . . . . . . . . . . . . . . . . . 9

3.5 Fractional-step formulation ........................... 9

4 The level set method 10

4.1 The level set equation . . . . . . . . . . . . . . . . . . . . . 10

4.2 Discontinuous gradient pressure functions . . . . . . . . . . . . . . . . 11

5 Numerical results $\quad 15$

5.1 Sloshing of two fluids in a square covered cavity . . . . . . . . . . . . . . 15

5.2 Jet buckling problem . . . . . . . . . . . . . . . . . . . . 17

6 Conclusions 21

$\begin{array}{ll}\text { References } & 28 \\ 28\end{array}$

\section{Introduction}

The numerical simulation of moving interfaces involved in two-fluid flow problems is an important topic in many industrial processes and physical situations. Dam break, sloshing in tanks, shallow waters, mould filling or inkjet analyses, are recurrent applications that involve the treatment of a surface evolution problem.

Viscoelastic fluid flows are present in several industrial processes involving paints, plastics, food or adhesives, but also in geophysical applications such as mud flows or avalanches. Accurate modelling of this type of flows can have a critical role in the optimization of the operational parameters of different processes or in the prediction of physical phenomena.

The classification of the methods used for free surface and two-fluid flows is not an easy task, mainly because of the wide range of numerical schemes that exist. However, one of the most general classifications depends on the nature of the mesh used, which can be fixed or not. In the present work we use a fixed mesh approach based on the level set method [1], which has been successfully used to validate experimental results that involve an interface evolution both in Newtonian and non-Newtonian fluids (see for example the review of Cruchaga et al. [2]). In the context of viscoelastic fluids the level set method has been used in the works of Yu et al. [3] and Pillapakkam and Singh [4]. Another common possibility is to use the volume of fluid approach (VOF) to evaluate the position of the moving interface, as in the work of Bonito et al. [5] where in addition, the authors use the SLIC post-processing algorithm (Simple Linear Interface Calculation) [6] to reduce numerical diffusion. The GENSMAC method (GENeralized Simplified Marker-And- Cell) [7] and its three-dimensional extension GENSMAC3D [8] is another possibility to follow the interface as shown in the works of Tomé et al. [9] and Figueiredo et al. [10]. See the work of McKee et al. [11] for a comprehensive review of the Marker and Cell (MAC) method.

The numerical solution of incompressible flows involving more than one fluid is one of the most challenging tasks in computational fluid dynamics [12]. The problems of tracing the interfaces and the discontinuity in the unknowns (velocity-stress-pressure) generated by the different material properties of the fluids on a time dependent domain are hard numerical tasks, which requires a great number of numerical ingredients in order to reach an accurate solution for this physical phenomenon. When the interface cuts an element, 
the discontinuity in the material properties leads to discontinuities in the gradients of the unknowns, which cannot be captured using standard interpolation functions. For instance, for two different density fluids at rest, the interpolation errors in pressure give rise to spurious velocities, which in turn give rise to spurious values in the elastic stresses. The combination of these errors causes the solution to lose physical sense. Also, viscosity discontinuities can lead to discontinuous velocity gradients that give rise to spurious stress and pressure values. Enriched methods add degrees of freedom at elements cut by the interface in order to reduce interpolation errors. Minev et al. [12] use a local enrichment of the bases for the velocity and pressure fields in a finite element formulation, which is used in the elements cut by the interface to improve the mass conservation properties; Newtonian fluids using the $P 2-P 1$ Taylor-Hood element are considered in this article. Chessa and Belytschko [13] use an enriched method based on the extended finite element method (XFEM), which is able to treat the material discontinuities. Coppola and Codina [14] propose a very easy-to-implement solution based on enriching the pressure shape functions on the elements cut by the interface to improve the ability to simulate the behavior of fluids with different densities under a gravitational force. The enrichment is used to enable the pressure gradient to be discontinuous at the interface, which could otherwise not be captured by the standard finite element shape functions and which can also be condensed prior to matrix assembly, thereby maintaining the number of degrees of freedom of the original problem.

The level set method may fail to preserve the total mass in applications that involve the interaction of immiscible fluids. A large number of publications exist in this context [15, 16, 17, 18]. A known approach to improve mass conservation properties of a level set method involves solving a continuity equation for the volume fraction of one phase of the problem. In this context hybrid level set/VOF methods [19] have been designed with important improvements in the mass conservation properties. Minev et al. [12] show that using local enrichment of the bases for the velocity and the pressure unknowns contributes to improving the mass conservation properties in the case of numerical simulations involving the effect of surface tension in Newtonian fluids.

The viscoelastic fluid flow problem presents several numerical difficulties, particularly for the spatial approximation. On the one hand, the finite element interpolation used must satisfy two inf-sup conditions, the same ones appearing in the Stokes or the Navier-Stokes problem written in a three field formulation $[20,21]$. On the other hand, the convective term in the momentum and in the constitutive equations requires the use of methods to deal with convection related instabilities. Many algorithms have been developed to solve this problem: see for example the works $[22,23,24]$ in the finite element context, and $[25,26]$ for the finite volume method. In this work we use a finite element variational multiscale formulation proposed by Castillo and Codina in [27]. In the present context, multiscale refers to the scales of the unknown that can be resolved by the numerical approximation and those that cannot. This discretization in space shows excellent stability properties, permitting in particular the use of equal interpolation spaces for all variables.

The inability to simulate viscoelastic flows at high Weissenberg numbers is currently one of the major challenges in computational rheology in the past few years and is known as the High Weissenberg Number Problem (HWNP). The log-conformation formulation for the elastic stress tensor proposed by Fattal and Kupfermann [28] is a common approach to solve highly elastic fluids and has been used for the free surface evolution problem successfully with different numerical methods $[9,30]$. Continuation methods are another numerical tool to increase the Weissenberg number limit that can be solved by a standard formulation, as shown in the work of Howell [31]. For the treatment of local oscillations, discontinuity-capturing techniques 
have proved to produce good results in [32, 27]. In this work we use a standard formulation for the elastic stress tensor with an additional discontinuity-capturing technique based on the orthogonal projection of the elastic stress tensor gradient as proposed in [27]. The formulation allows one to solve the jet buckling problem in the range of Weissenberg number $0<$ We $<100$ using the Oldroyd-B model.

We present here two numerical simulations to illustrate the capability of our finite element formulation to simulate moving interfaces using the Oldroyd-B and the Giesekus constitutive models. The first example consists in the resolution of a sloshing case that involves the interaction between a Giesekus fluid and a Newtonian one. Secondly, the jet buckling problem is solved to compare the results using the proposed methodology with previously published works using the Oldroyd-B constitutive model. Both examples are solved both in the two-dimensional and in the three-dimensional case.

The work is organized as follows. In Section 2 we introduce the continuous viscoelastic fluid flow problem. Section 3 is devoted to its numerical approximation with the Galerkin approximation and the stabilized formulations used. In Section 4, the level set method and the enriched pressure shape functions are presented. Numerical results are shown in Section 5 and, finally, conclusions are drawn in Section 6.

\section{The viscoelastic flow problem}

\subsection{Boundary value problem}

To simulate the transient incompressible and isothermal flow of viscoelastic fluids, one needs to solve the momentum balance equation, the continuity equation and a constitutive equation that defines the viscoelastic contribution of the fluid.

The conservation equations for momentum and mass may be expressed for each fluid as:

$$
\begin{aligned}
\rho_{i} \frac{\partial \boldsymbol{u}_{i}}{\partial t}+\rho_{i} \boldsymbol{u}_{i} \cdot \nabla \boldsymbol{u}_{i}-\nabla \cdot \boldsymbol{T}_{i}+\nabla p_{i} & \left.=\boldsymbol{f}_{i} \quad \text { in } \Omega_{i}, t \in\right] 0, t_{\mathrm{f}}[ \\
\nabla \cdot \boldsymbol{u}_{i} & \left.=0 \quad \text { in } \Omega_{i}, t \in\right] 0, t_{\mathrm{f}}[
\end{aligned}
$$

where $\Omega_{i}$ is the computational domain of $\mathbb{R}^{d}$ occupied by the fluid $i=1$ or $2, d=2$ or 3 being the space dimensions. The whole domain $\Omega$ is defined as $\left.\Omega=\Omega_{1} \cup \Omega_{2},\right] 0, t_{\mathrm{f}}[$ is the time interval in which the problem is solved, $\rho_{i}$ denotes the density, $\left.p_{i}: \Omega_{i} \times\right] 0, t_{\mathrm{f}}\left[\longrightarrow \mathbb{R}\right.$ the pressure, $\left.\boldsymbol{u}_{i}: \Omega_{i} \times\right] 0, t_{\mathrm{f}}\left[\longrightarrow \mathbb{R}^{d}\right.$ the velocity field, $\boldsymbol{f}_{i}: \Omega_{i} \rightarrow \mathbb{R}^{d}$ the force vector, taken as the gravity force $\rho_{i} \boldsymbol{g}$, and $\left.\boldsymbol{T}_{i}: \Omega_{i} \times\right] 0, t_{\mathrm{f}}\left[\longrightarrow \mathbb{R}^{d} \otimes \mathbb{R}^{d}\right.$ is the deviatoric extra stress tensor, which can be defined in terms of a viscous and a viscoelastic or elastic contribution as:

$$
\boldsymbol{T}_{i}=2 \beta_{i} \eta_{0, i} \nabla^{s} \boldsymbol{u}_{i}+\boldsymbol{\sigma}_{i}
$$

where $\nabla^{s}$ is the symmetric gradient operator, $\beta_{i} \in[0,1]$ is a real parameter to define the amount of viscous or solvent viscosity $\eta_{s, i}=\beta_{i} \eta_{0, i}$ and elastic or polymeric viscosity $\eta_{p, i}=\left(1-\beta_{i}\right) \eta_{0, i}$ in the fluid. For viscoelastic fluids, the problem is incomplete without the definition of a constitutive equation for the elastic part of the extra stress tensor $\left(\boldsymbol{\sigma}_{i}\right)$. A large variety of approaches exist to define it (see [33,34] for a complete description). In this work, we use the Oldroyd-B and the Giesekus constitutive models to define the fluid. 
Both models can be defined using the equation

$$
\begin{aligned}
\frac{\lambda_{i}}{2 \eta_{0, i}} \frac{\partial \boldsymbol{\sigma}_{i}}{\partial t}-\left(1-\beta_{i}\right) \nabla^{s} \boldsymbol{u}_{i}+\frac{\lambda_{i}}{2 \eta_{0, i}}\left(\boldsymbol{u}_{i} \cdot \nabla \boldsymbol{\sigma}_{i}-\boldsymbol{\sigma}_{i} \cdot \nabla \boldsymbol{u}_{i}-\left(\nabla \boldsymbol{u}_{i}\right)^{T} \cdot \boldsymbol{\sigma}_{i}\right) & \\
& \left.+\frac{1}{2 \eta_{0, i}}\left(1+h\left(\boldsymbol{\sigma}_{i}\right)\right) \cdot \boldsymbol{\sigma}_{i}=\mathbf{0} \quad \text { in } \Omega, t \in\right] 0, t_{\mathrm{f}}[.
\end{aligned}
$$

The Giesekus model is obtained replacing $h\left(\sigma_{i}\right)=\frac{\varepsilon_{i} \lambda_{i}}{\eta_{p, i}} \sigma_{i}$ in (4), where $\lambda_{i}$ is the relaxation time and $\varepsilon_{i}$ the mobility factor. When $\varepsilon_{i}=0$, the Giesekus model reduces to the Oldroyd-B rheological model. Note that $h(\boldsymbol{\sigma})$ is a tensor, and thus $1+h$ should be understood as identity plus $h$.

The non-linear term $\left(\boldsymbol{\sigma}_{i} \cdot \boldsymbol{\sigma}_{i}\right)$ of the Giesekus model enables simply qualitative descriptions of a number of well-known properties of viscoelastic fluids, namely, shear thinning, the non-zero second normal stress coefficient, and stress overshoot in transient shear flows [35].

To avoid excessive nomenclature we will omit the subindex $i$ that defines each fluid in the exposition of the methods, unless the expression needs explicitly this definition.

Let us introduce some notation used in the next subsections. Calling $\boldsymbol{U}=[\boldsymbol{u}, \boldsymbol{\sigma}, p], \boldsymbol{F}=[\boldsymbol{f}, 0,0]$ and defining

$$
\mathcal{L}(\hat{\boldsymbol{u}}, \hat{\boldsymbol{\sigma}} ; \boldsymbol{U}):=\left(\begin{array}{c}
\rho \hat{\boldsymbol{u}} \cdot \nabla \boldsymbol{u}-2 \beta \eta_{0} \nabla \cdot\left(\nabla^{s} \boldsymbol{u}\right)-\nabla \cdot \boldsymbol{\sigma}+\nabla p \\
\nabla \cdot \boldsymbol{u} \\
-(1-\beta) \nabla^{s} \boldsymbol{u}+\frac{\lambda}{2 \eta_{0}}\left(\hat{\boldsymbol{u}} \cdot \nabla \boldsymbol{\sigma}-\boldsymbol{\sigma} \cdot \nabla \hat{\boldsymbol{u}}-(\nabla \hat{\boldsymbol{u}})^{T} \cdot \boldsymbol{\sigma}\right)+\frac{1}{2 \eta_{0}}(1+h(\hat{\boldsymbol{\sigma}})) \cdot \boldsymbol{\sigma}
\end{array}\right)
$$

and

$$
\mathcal{D}_{t}(\boldsymbol{U}):=\left(\begin{array}{c}
\rho \frac{\partial \boldsymbol{u}}{\partial t} \\
0 \\
\frac{\lambda}{2 \eta_{0}} \frac{\partial \boldsymbol{\sigma}}{\partial t}
\end{array}\right)
$$

we may write (1), (2) and (4) using the definition (3) as:

$$
\mathcal{D}_{t}(\boldsymbol{U})+\mathcal{L}(\boldsymbol{u}, \boldsymbol{\sigma} ; \boldsymbol{U})=\boldsymbol{F} .
$$

These equations are a mixed parabolic-hyperbolic system. Additionally to the three equations defined by (5), the problem needs initial and boundary conditions both in the velocity and the elastic stress fields to close the problem. In principle the elastic stresses can be fixed only on the inflow part of the boundary $\Gamma_{\text {in }}=\{\boldsymbol{x} \in \partial \Omega \mid(\boldsymbol{u} \cdot \boldsymbol{n})(\boldsymbol{x})<0\}$, where $\boldsymbol{n}$ is the outward unit normal vector to $\partial \Omega$. For a complete description of the mathematical structure of the problem and the boundary conditions required, see for example the work of Fernández-Cara et al. [36]. For the sake of conciseness, the boundary conditions for the velocity will be taken of homogeneous Dirichlet type, even if zero traction boundary conditions will also be used in the numerical simulations. Note that pressure will be determined up to a constant.

\subsection{The variational form}

Let us introduce some notation in order to write the weak form of the problem. The space of square integrable functions in a domain $\omega$ is denoted by $L^{2}(\omega)$, and the space of functions whose distributional derivatives of order up to $m \geq 0$ (integer) belong to $L^{2}(\omega)$ by $H^{m}(\omega)$. The space $H_{0}^{m}(\omega)$ consists of functions in $H^{1}(\omega)$ vanishing on $\partial \omega$. The topological dual of $H_{0}^{1}(\Omega)$ is denoted by $H^{-1}(\Omega)$, the duality 
pairing by $\langle\cdot, \cdot\rangle$, and the $L^{2}$ inner product in $\Omega$ (for scalar, vectors and tensors) is denoted by $(\cdot, \cdot)$. The integral of the product of two functions in a domain $\omega$ is denoted as $\langle\cdot, \cdot\rangle_{\omega}$.

Let $\Upsilon=H^{1}(\Omega)_{\mathrm{sym}}^{d \times d}$ (symmetric second order tensors with components in $H^{1}(\Omega)$ ), $\mathcal{V}=H_{0}^{1}(\Omega)^{d}$ and $\mathcal{Q}=L^{2}(\Omega) / \mathbb{R}$, be the spaces where we may seek the elastic stress, the velocity and the pressure, respectively, for each fixed time $t$. Let also $\boldsymbol{U}=[\boldsymbol{u}, p, \boldsymbol{\sigma}]$. The weak form of the problem is obtained by testing (5) against an arbitrary test function $\boldsymbol{V}=[\boldsymbol{v}, q, \boldsymbol{\tau}]$ with appropriate regularity. It can be written as: find

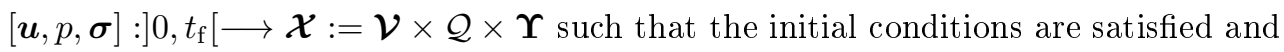

$$
\begin{aligned}
\left(\rho \frac{\partial \boldsymbol{u}}{\partial t}, \boldsymbol{v}\right)+2\left(\beta \eta_{0} \nabla^{s} \boldsymbol{u}, \nabla^{s} \boldsymbol{v}\right)+\langle\rho \boldsymbol{u} \cdot \nabla \boldsymbol{u}, \boldsymbol{v}\rangle+\left(\boldsymbol{\sigma}, \nabla^{s} \boldsymbol{v}\right)-(p, \nabla \cdot \boldsymbol{v}) & =\langle\boldsymbol{f}, \boldsymbol{v}\rangle, \\
\left(\frac{\lambda}{2 \eta_{0}} \frac{\partial \boldsymbol{\sigma}}{\partial t}, \boldsymbol{\tau}\right)-\left((1-\beta) \nabla^{s} \boldsymbol{u}, \boldsymbol{\tau}\right)+\frac{\lambda}{2 \eta_{0}}((\boldsymbol{u} \cdot \nabla \boldsymbol{\sigma})=0 & \left.\left.\boldsymbol{\sigma} \cdot \nabla \boldsymbol{u}-(\nabla \boldsymbol{u})^{T} \cdot \boldsymbol{\sigma}\right), \boldsymbol{\tau}\right) \\
+\left(\frac{1}{2 \eta_{0}} \boldsymbol{\sigma}, \boldsymbol{\tau}\right)+\left(\frac{\lambda \varepsilon}{2(1-\beta) \eta_{0}^{2}} \boldsymbol{\sigma} \cdot \boldsymbol{\sigma}, \boldsymbol{\tau}\right) & =\mathbf{0}
\end{aligned}
$$

for all $\boldsymbol{V}=[\boldsymbol{v}, q, \boldsymbol{\tau}] \in \mathcal{X}$, where it is assumed that $\boldsymbol{f}$ is such that $\langle\boldsymbol{f}, \boldsymbol{v}\rangle$ is well defined. The last term of the constitutive equation appears only in the Giesekus model but it is always included to avoid more definitions.

In a compact form, the problem (6)-(8) can be written as:

$$
\left(\mathcal{D}_{t}(\boldsymbol{U}), \boldsymbol{V}\right)+B(\boldsymbol{u}, \boldsymbol{\sigma} ; \boldsymbol{U}, \boldsymbol{V})=\langle\boldsymbol{f}, \boldsymbol{v}\rangle
$$

for all $\boldsymbol{V} \in \mathcal{X}$, where

$$
\begin{aligned}
B(\hat{\boldsymbol{u}}, \hat{\boldsymbol{\sigma}} ; \boldsymbol{U}, \boldsymbol{V}) & =2\left(\beta \eta_{0} \nabla^{s} \boldsymbol{u}, \nabla^{s} \boldsymbol{v}\right)+\langle\rho \hat{\boldsymbol{u}} \cdot \nabla \boldsymbol{u}, \boldsymbol{v}\rangle+\left(\boldsymbol{\sigma}, \nabla^{s} \boldsymbol{v}\right)-(p, \nabla \cdot \boldsymbol{v})+(q, \nabla \cdot \boldsymbol{u}) \\
& -\left((1-\beta) \nabla^{s} \boldsymbol{u}, \boldsymbol{\tau}\right)+\frac{\lambda}{2 \eta_{0}}\left(\left(\hat{\boldsymbol{u}} \cdot \nabla \boldsymbol{\sigma}-\boldsymbol{\sigma} \cdot \nabla \hat{\boldsymbol{u}}-(\nabla \hat{\boldsymbol{u}})^{T} \cdot \boldsymbol{\sigma}\right), \boldsymbol{\tau}\right) \\
& +\left(\frac{1}{2 \eta_{0}} \boldsymbol{\sigma}, \boldsymbol{\tau}\right)+\left(\frac{\lambda \varepsilon}{2(1-\beta) \eta_{0}^{2}} \hat{\boldsymbol{\sigma}} \cdot \boldsymbol{\sigma}, \boldsymbol{\tau}\right) .
\end{aligned}
$$

It is understood that integrals involved in this expression extend either over $\Omega_{1}$ or over $\Omega_{2}$, according to the fluid that occupies a certain region, with its physical properties. Appropriate transmission conditions (continuity of velocity and of the normal component of the total stress) are ensured by writing the problem in this global form.

\section{Numerical approximation}

\subsection{Galerkin finite element discretization}

The standard Galerkin approximation for the variational problem can be performed by considering a finite element partition $\mathcal{T}_{h}$ of the domain $\Omega$. The diameter of an element domain $K \in \mathcal{T}_{h}$ is denoted by $h_{K}$ and the diameter of the element partition is defined by $h=\max \left\{h_{K} \mid K \in \mathcal{T}_{h}\right\}$. Under the above considerations, we can construct conforming finite elements spaces, $\mathcal{V}_{h} \subset \mathcal{V}, \mathcal{Q}_{h} \subset \mathcal{Q}$ and $\boldsymbol{\Upsilon}_{h} \subset \boldsymbol{\Upsilon}$ in the usual manner. If $\mathcal{X}_{h}=\mathcal{V}_{h} \times \mathcal{Q}_{h} \times \boldsymbol{\Upsilon}_{h}$, and $\boldsymbol{U}_{h}=\left[\boldsymbol{u}_{h}, p_{h}, \boldsymbol{\sigma}_{h}\right]$, the Galerkin finite element approximation consists in finding $\left.\boldsymbol{U}_{h}:\right] 0, t_{\mathrm{f}}\left[: \longrightarrow \mathcal{X}_{h}\right.$ such that:

$$
\left(\mathcal{D}_{t}\left(\boldsymbol{U}_{h}\right), \boldsymbol{V}_{h}\right)+B\left(\boldsymbol{u}_{h}, \boldsymbol{\sigma}_{h} ; \boldsymbol{U}_{h}, \boldsymbol{V}_{h}\right)=\left\langle\boldsymbol{f}, \boldsymbol{v}_{h}\right\rangle
$$


for all $\boldsymbol{V}_{h}=\left[\boldsymbol{v}_{h}, q_{h}, \boldsymbol{\tau}_{h}\right] \in \mathcal{X}_{h}$.

\subsection{Stabilized finite element formulation}

Choosing equal order approximations for $\boldsymbol{\sigma}, \boldsymbol{u}$ and $p$ does not yield a stable scheme. A possible remedy to this situation is to enrich the finite element spaces for the velocity and the stress in order to satisfy the two compatibility conditions associated with the viscoelastic problem (see for example [37] and references therein). Another possibility is to use stabilized formulations permitting any interpolation of the variables, which is the approach we use in this paper. In general, a stabilized formulation consists of replacing $B$ in (11) by another semi-linear form $B_{h}$, possibly mesh dependent, with better stability properties.

Stabilized finite element methods consist in modifying the discrete Galerkin formulation of the problem by adding terms designed to enhance stability without upsetting accuracy. In this work we use the split orthogonal sub-grid (Split OSGS) method proposed in [27] for the stationary viscoelastic problem using the Oldroyd-B constitutive model, and tested in transient problems in [38] in the context of fractional step methods. The theoretical foundations of the method, applied to linearized Newtonian flows, are presented in [39].

The method consists in replacing (11) by the following problem: find $\left.\boldsymbol{U}_{h}:\right] 0, t_{\mathrm{f}}\left[\rightarrow \boldsymbol{\mathcal { X }}_{h}\right.$, such that

$$
\left(\mathcal{D}_{t}\left(\boldsymbol{U}_{h}\right), \boldsymbol{V}_{h}\right)+B\left(\boldsymbol{u}_{h}, \boldsymbol{\sigma}_{h} ; \boldsymbol{U}_{h}, \boldsymbol{V}_{h}\right)+S_{1}^{\perp}\left(\boldsymbol{u}_{h} ; \boldsymbol{U}_{h}, \boldsymbol{V}_{h}\right)+S_{2}^{\perp}\left(\boldsymbol{U}_{h}, \boldsymbol{V}_{h}\right)+S_{3}^{\perp}\left(\boldsymbol{u}_{h}, \boldsymbol{\sigma}_{h} ; \boldsymbol{U}_{h}, \boldsymbol{V}_{h}\right)=\left\langle\boldsymbol{f}, \boldsymbol{v}_{h}\right\rangle
$$

for all $\boldsymbol{V}_{h} \in \mathcal{X}_{h}$, where

$$
\begin{aligned}
S_{1}^{\perp}\left(\hat{\boldsymbol{u}}_{h} ; \boldsymbol{U}_{h}, \boldsymbol{V}_{h}\right) & =\sum_{K} \alpha_{1}\left\langle P_{h}^{\perp}\left[\rho \hat{\boldsymbol{u}}_{h} \cdot \nabla \boldsymbol{u}_{h}\right], \rho \hat{\boldsymbol{u}}_{h} \cdot \nabla \boldsymbol{v}_{h}\right\rangle_{K}+\sum_{K} \alpha_{1}\left\langle P_{h}^{\perp}\left[\nabla p_{h}-\rho \boldsymbol{g}\right], \nabla q_{h}\right\rangle_{K} \\
& +(1-\beta) \sum_{K} \alpha_{1}\left\langle P_{h}^{\perp}\left[\nabla \cdot \boldsymbol{\sigma}_{h}\right], \nabla \cdot \boldsymbol{\tau}_{h}\right\rangle_{K}, \\
S_{2}^{\perp}\left(\boldsymbol{U}_{h}, \boldsymbol{V}_{h}\right) & =\sum_{K} \alpha_{2}\left\langle P_{h}^{\perp}\left[\nabla \cdot \boldsymbol{u}_{h}\right], \nabla \cdot \boldsymbol{v}_{h}\right\rangle_{K}, \\
S_{3}^{\perp}\left(\hat{\boldsymbol{u}}_{h}, \hat{\boldsymbol{\sigma}}_{h} ; \boldsymbol{U}_{h}, \boldsymbol{V}_{h}\right) & =\sum_{K} \alpha_{3}\left\langle P_{h}^{\perp}\left[\boldsymbol{R}_{\sigma, h}\left(\hat{\boldsymbol{u}}_{h}, \hat{\boldsymbol{\sigma}}_{h} ; \boldsymbol{U}_{h}\right)\right],\right. \\
& \left.-\frac{1}{2 \eta_{0}} h\left(\hat{\boldsymbol{\sigma}}_{h}\right)^{T} \boldsymbol{\tau}_{h}-\nabla^{s} \boldsymbol{v}_{h}+\frac{\lambda}{2 \eta_{0}}\left(\hat{\boldsymbol{u}}_{h} \cdot \nabla \boldsymbol{\tau}_{h}-\boldsymbol{\tau}_{h} \cdot\left(\nabla \hat{\boldsymbol{u}}_{h}\right)^{T}-\nabla \hat{\boldsymbol{u}}_{h} \cdot \boldsymbol{\tau}_{h}\right)\right\rangle_{K}
\end{aligned}
$$

where $P_{h}^{\perp}=I-P_{h}$ is the orthogonal projection, with $P_{h}$ the $L^{2}$ projection onto the appropriate finite element space. In the last expression, $\boldsymbol{R}_{\sigma, h}$ represents the residual of the constitutive equation without the local temporal derivative and without the term $\frac{1}{2 \eta_{0}} \boldsymbol{\sigma}_{h}$ :

$$
\boldsymbol{R}_{\sigma, h}\left(\hat{\boldsymbol{u}}_{h}, \hat{\boldsymbol{\sigma}}_{h} ; \boldsymbol{U}_{h}\right)=-(1-\beta) \nabla^{s} \boldsymbol{u}_{h}+\frac{\lambda}{2 \eta_{0}}\left(\hat{\boldsymbol{u}}_{h} \cdot \nabla \boldsymbol{\sigma}_{h}-\boldsymbol{\sigma}_{h} \cdot \nabla \hat{\boldsymbol{u}}_{h}-\left(\nabla \hat{\boldsymbol{u}}_{h}\right)^{T} \cdot \boldsymbol{\sigma}_{h}\right)+\frac{1}{2 \eta_{0}} h\left(\hat{\boldsymbol{\sigma}}_{h}\right) \cdot \boldsymbol{\sigma}_{h} .
$$

The orthogonal projection of all these terms converges to zero at the optimal rate allowed by the finite element interpolation. In particular, this is why the temporal term has been neglected even if it is not exactly zero for non-constant physical properties (note that the orthogonal projection of the temporal derivative of $\boldsymbol{\sigma}_{h}$ is exactly zero). For the same reason, the last term in (13) could be neglected.

The parameters $\alpha_{j}, j=1,2,3$ represent the components of the stabilizing parameter matrix presented in 
[27], computed within each element $K$ as

$$
\begin{aligned}
& \alpha_{1}=\left[c_{1} \frac{\eta_{0}}{h_{1}^{2}}+c_{2} \frac{\rho\left|\boldsymbol{u}_{h}\right|}{h_{2}}\right]^{-1}, \quad \alpha_{2}=\frac{h_{1}^{2}}{c_{1} \alpha_{1}} \\
& \alpha_{3}=\left[c_{3} \frac{1}{2 \eta_{0}}+c_{4}\left(\frac{\lambda}{2 \eta_{0}} \frac{\left|\boldsymbol{u}_{h}\right|}{h_{2}}+\frac{\lambda}{\eta_{0}}\left|\nabla \boldsymbol{u}_{h}\right|\right)\right]^{-1} .
\end{aligned}
$$

In these expressions, $h_{1}$ corresponds to a characteristic element length calculated as the square root of the element area in $2 \mathrm{D}$ case and the cubic root of the element volume in $3 \mathrm{D}$, and $h_{2}$ corresponds to another characteristic length calculated as the element length in the streamline direction. The constants $c_{j}, j=$ $1,2,3,4$ are algorithmic parameters in the formulation. The values used in this work (for linear elements) are $c_{1}=12.0, c_{2}=2.0, c_{3}=4.0$ and $c_{4}=1.0$. For higher order elements, the characteristic lengths $h_{1}$ and $h_{2}$ should be divided respectively by $r^{2}$ and $r, r$ being the order of the finite element interpolation. The stabilization parameters need to be computed at each integration point, and thus the physical properties to be employed in their evaluation depend on the fluid (1 or 2$)$ that occupies each point.

The stabilizing mechanisms introduced by terms $S_{1}^{\perp}, S_{2}^{\perp}$ and $S_{3}^{\perp}$ are the following. The first component of the $S_{1}^{\perp}$ gives control on the convective term, the second component gives control on the pressure gradient and includes the gravity force term, and the third term gives control on the divergence of the viscoelastic stress. The term $S_{2}^{\perp}$ is not always necessary but in some cases it improves stability of the problem. Finally, the term $S_{3}^{\perp}$ ensures stability of the constitutive equation. Note that some of the components of this last term are the convective-convective term of the viscoelastic stress tensor and an equivalent EVSS-structure component (see [40]) obtained when testing the orthogonal component of the first term in (13) with $\nabla^{s} \boldsymbol{v}_{h}$, among other cross local inner-product terms.

The addition of $S_{1}^{\perp}, S_{2}^{\perp}$ and $S_{3}^{\perp}$ permit the approximation of convection-dominated problems both in velocity and in stress, and the implementation of equal order interpolation for all the unknowns (see [27] for more details on the stabilized formulation used).

\subsection{Discontinuity-Capturing technique}

The previously described stabilized finite element formulation yields a globally stable solution, i.e., norms of the unknowns over the whole domain are bounded. However, if the solution displays large gradients, local oscillations may still remain.

In the case of viscoelastic flow problems, local instabilities or very high gradients in the pressure and in the viscoelastic stress components can be found when the fluid flow finds an abrupt change in the geometry. In order to overcome these instabilities, we use the discontinuity capturing term proposed in [27] based on the orthogonal projection of the elastic stress tensor gradient. The implementation is very easy and standard for any type of formulation, and consists in adding to the left hand side of the constitutive equation the following term:

$$
\sum_{K}\left\langle\kappa_{\sigma} \nabla \boldsymbol{\sigma}_{h}, \nabla \boldsymbol{\tau}_{h}\right\rangle_{K}
$$

where the diffusion coefficient $\kappa_{\sigma}$ is defined as

$$
\kappa_{\sigma}=\left(C_{a} h_{1}\left|\boldsymbol{u}_{h}\right|+C_{b} h_{1}^{2}\left|\nabla \boldsymbol{u}_{h}\right|\right) \frac{\left|P_{h}^{\perp}\left(\nabla \boldsymbol{\sigma}_{h}\right)\right|}{\left|\nabla \boldsymbol{\sigma}_{h}\right|} .
$$

Note that this term converges to zero at the optimal rate permitted by the finite element interpolation, at 
least when the solution is smooth. The values used in this work for the constants appearing in the numerical diffusion are $C_{a}=1.0$ and $C_{b}=1.0$, but they can be tuned depending on the properties of the particular flow.

\subsection{Monolithic time discretization}

Let us explain how to discretize in time problem (11) using a monolithic approach. Any time discretization is possible, although for the sake of conciseness we will restrict the explanation to the classical backwarddifference (BDF) approximation, even if in some parts of the work we use the Crank Nicolson scheme.

The time interval $] 0, t_{\mathrm{f}}$ [ can be partitioned into time steps of size $\delta t$, which are considered to be constant for simplicity. Let us use a superscript to denote the time step counter. The BDF approximation to the time derivative of a time dependent function $y(t)$ of order $k=1,2, .$. , is given by $\delta_{k} y^{n+1} / \delta t$, with

$$
\delta_{k} y^{n+1}=\frac{1}{\gamma_{k}}\left(y^{n+1}-\sum_{i=0}^{k-1} \varphi_{k}^{i} y^{n-i}\right)
$$

where $\gamma_{k}$ and $\varphi_{k}^{i}$ are numerical parameters. In particular, for the cases $k=1$ and 2 (BDF1 and BDF2) we have:

$$
\begin{aligned}
& \delta_{1} y^{n+1}=y^{n+1}-y^{n}, \\
& \delta_{2} y^{n+1}=\frac{3}{2}\left(y^{n+1}-\frac{4}{3} y^{n}+\frac{1}{3} y^{n-1}\right) .
\end{aligned}
$$

We will also use the extrapolation of order 1 , defined as $\hat{y}_{1}^{n+1}=y^{n}+\mathcal{O}(\delta t)$.

To simplify the description of the time integration, let us consider the Galerkin method. Using a BDF scheme, the time discretization of problem (11) can be written, in expanded form, as: given the initial conditions, for $n=1,2, \ldots$, find $\left[\boldsymbol{u}_{h}^{n+1}, p_{h}^{n+1}, \boldsymbol{\sigma}_{h}^{n+1}\right] \in \mathcal{X}_{h}$, such that:

$$
\begin{aligned}
& \left(\rho \frac{\delta_{k} \boldsymbol{u}_{h}}{\delta t}, \boldsymbol{v}_{h}\right)+2\left(\beta \eta_{0} \nabla^{s} \boldsymbol{u}_{h}^{n+1}, \nabla^{s} \boldsymbol{v}_{h}\right)+\left\langle\rho \boldsymbol{u}_{h}^{n+1} \cdot \nabla \boldsymbol{u}_{h}^{n+1}, \boldsymbol{v}_{h}\right\rangle+\left(\boldsymbol{\sigma}_{h}^{n+1}, \nabla^{s} \boldsymbol{v}_{h}\right) \\
& \quad-\left(p_{h}^{n+1}, \nabla \cdot \boldsymbol{v}_{h}\right)=\left\langle\boldsymbol{f}_{h}^{n+1}, \boldsymbol{v}_{h}\right\rangle, \\
& \left(\frac{\lambda}{2}, \nabla \cdot \boldsymbol{u}_{h}^{n+1}\right)=0 \\
& \left(\frac{\lambda}{2 \eta_{0}} \frac{\delta_{k} \boldsymbol{\sigma}_{h}^{n+1}}{\delta t}, \boldsymbol{\tau}_{h}\right)+\left(\frac{1}{2 \eta_{0}} \boldsymbol{\sigma}_{h}^{n+1}, \boldsymbol{\tau}_{h}\right)-\left((1-\beta) \nabla^{s} \boldsymbol{u}_{h}^{n+1}, \boldsymbol{\tau}_{h}\right) \\
& \quad+\frac{\lambda}{2 \eta_{0}}\left(\left(\boldsymbol{u}_{h}^{n+1} \cdot \nabla \boldsymbol{\sigma}_{h}^{n+1}-\boldsymbol{\sigma}_{h}^{n+1} \cdot \nabla \boldsymbol{u}_{h}^{n+1}-\left(\nabla \boldsymbol{u}_{h}^{n+1}\right)^{T} \cdot \boldsymbol{\sigma}_{h}^{n+1}\right), \boldsymbol{\tau}_{h}\right) \\
& \quad+\left(\frac{\lambda \alpha}{2(1-\beta) \eta_{0}^{2}} \boldsymbol{\sigma}_{h}^{n+1} \cdot \boldsymbol{\sigma}_{h}^{n+1}, \boldsymbol{\tau}_{h}\right)=0
\end{aligned}
$$

for all $\boldsymbol{V}_{h} \in \mathcal{X}_{h}$. The fully discrete stabilized problem can be defined in the same way (see [38]).

\subsection{Fractional-step formulation}

The monolithic resolution of the system of equations that is obtained after discretization of the continuous problem is the most straightforward option. However, solving this system is computationally very expensive, specially in 3D. In some of the numerical examples, we have uses a fractional step approach to solve the two immiscible fluid flow problem. This fractional step algorithm was proposed in [38] and gives second order 
accuracy in time, even if a third order scheme is also proposed in this article. For a review of fractional step schemes designed at the pure algebraic level, see [41].

Problem (14)-(16) can be written in the following algebraic form:

$$
\begin{aligned}
M_{\boldsymbol{u}} \frac{\delta_{k}}{\delta t} \mathbf{U}^{n+1}+K_{\boldsymbol{u}}\left(\mathbf{U}^{n+1}\right) \mathbf{U}^{n+1}+G \mathrm{P}^{n+1}-D_{\sigma} \boldsymbol{\Sigma}^{n+1} & =F^{n+1}, \\
D \mathbf{U}^{n+1} & =\mathbf{0}, \\
M_{\boldsymbol{\sigma}} \frac{\delta_{k}}{\delta t} \boldsymbol{\Sigma}^{n+1}+K_{\boldsymbol{\sigma}}\left(\mathbf{U}^{n+1}\right) \boldsymbol{\Sigma}^{n+1}-S \mathbf{U}^{n+1} & =\mathbf{0}
\end{aligned}
$$

where $\mathbf{U}, \mathrm{P}$ and $\boldsymbol{\Sigma}$ are arrays of the nodal unknowns for $\boldsymbol{u}, p$ and $\boldsymbol{\sigma} . K_{\boldsymbol{u}}$ and $K_{\boldsymbol{\sigma}}$ are the stiffness matrices of the momentum and constitutive equation, $G$ represent the gradient operator, $D$ is the divergence of the velocity, $D_{\sigma}$ the divergence of the elastic stress tensor and $S$ the symmetrical part of the velocity gradient. $M_{u}$ and $M_{\sigma}$ represent the mass matrices of the momentum and the constitutive equation. We are using the same notation used in [38] to facilitate the explanation. Additional terms coming from stabilization can be easily treated, as explained in this article.

Roughly speaking, a fractional step method consists in the resolution of the full problem in different algorithmic steps that permit the calculation of all the variables in a sequential way. The different steps can be separated in steps where intermediate values are obtained, denoted by $\tilde{(}^{n+1}$, and steps where the final values ()$^{n+1}$ are determined correcting the intermediate values.

The fractional step algorithm used in this work is of formal second order accuracy in time and can be written in matrix form in terms of intermediate and final steps, as it is shown in algorithms (1) and (2) (see [38] for more details). The extrapolated first order values $\hat{\boldsymbol{\Sigma}}_{1}^{n+1}$ and $\hat{\mathrm{P}}_{1}^{n+1}$ allow us to obtain the second order accuracy of the fractional step algorithm.

Algorithm 1 Fractional step approach: intermediate steps.

1. Intermediate velocity using the pressure and the elastic stress values extrapolated:

$$
\frac{\delta_{k}}{\delta t} M_{\boldsymbol{u}} \tilde{\mathbf{U}}^{n+1}+K_{\boldsymbol{u}}\left(\tilde{\mathbf{U}}^{n+1}\right) \tilde{\mathbf{U}}^{n+1}=\boldsymbol{f}-G \hat{\mathrm{P}}_{1}^{n+1}+D_{\boldsymbol{\sigma}} \hat{\boldsymbol{\Sigma}}_{1}^{n+1} \rightarrow \tilde{\mathbf{U}}^{n+1}
$$

2. Intermediate elastic stress values using the intermediate velocity:

$$
\frac{\delta_{k}}{\delta t} M_{\boldsymbol{\sigma}} \tilde{\boldsymbol{\Sigma}}^{n+1}+K_{\boldsymbol{\sigma}}\left(\tilde{\mathbf{U}}^{n+1}\right) \tilde{\boldsymbol{\Sigma}}^{n+1}-S \tilde{\mathbf{U}}^{n+1}=\boldsymbol{f}_{\boldsymbol{c}} \rightarrow \tilde{\boldsymbol{\Sigma}}^{n+1}
$$

3. Intermediate pressure calculation using the intermediate velocity and elastic stress:

$$
-D \tilde{\mathbf{U}}^{n+1}+\gamma_{k} \delta t D M_{\boldsymbol{u}}^{-1} G\left(\tilde{\mathrm{P}}^{n+1}-\hat{\mathrm{P}}_{1}^{n+1}\right)-\gamma_{k} \delta t D M_{\boldsymbol{u}}^{-1} D_{\sigma}\left(\tilde{\boldsymbol{\Sigma}}^{n+1}-\hat{\boldsymbol{\Sigma}}_{1}^{n+1}\right)=0 \rightarrow \tilde{\mathrm{P}}^{n+1}
$$

The stabilized version can be written in the same way as the Galerkin formulation. For more details see [38] where the fractional step algorithm for viscoelastic fluids used in this paper was developed.

\section{The level set method}

\subsection{The level set equation}

The level set method is based on the pure advection of a smooth function, say $\phi(\boldsymbol{x}, t)$, over the whole computational domain $\Omega=\Omega_{1} \cup \Omega_{2}$. This function defines the position of the front or the interface, by the 
Algorithm 2 Fractional step approach: final steps.

4. Velocity correction:

$$
\frac{1}{\gamma_{k} \delta t} M_{\boldsymbol{u}}\left(\mathbf{U}^{n+1}-\tilde{\mathbf{U}}^{n+1}\right)+G\left(\tilde{\mathrm{P}}^{n+1}-\hat{\mathrm{P}}_{1}^{n+1}\right)-D_{\sigma}\left(\tilde{\boldsymbol{\Sigma}}^{n+1}-\hat{\mathbf{\Sigma}}_{1}^{n+1}\right)=\mathbf{0} \rightarrow \mathbf{U}^{n+1}
$$

5. Elastic stress correction:

$$
\frac{1}{\gamma_{k} \delta t} M_{\boldsymbol{\sigma}}\left(\boldsymbol{\Sigma}^{n+1}-\tilde{\mathbf{\Sigma}}^{n+1}\right)-S\left(\mathbf{U}^{n+1}-\tilde{\mathbf{U}}^{n+1}\right)=\mathbf{0} \rightarrow \boldsymbol{\Sigma}^{n+1}
$$

6. Pressure correction (formally):

$$
\mathrm{P}^{n+1}=\tilde{\mathrm{P}}^{n+1} \rightarrow \mathrm{P}^{n+1}
$$

isovalue $\phi(\boldsymbol{x}, t)=\phi_{c}$ where $\phi_{c}$ is a critical value defined a priori; in this work we have taken $\phi_{c}=0$. Thereby, the domain of the first fluid $\left(\Omega_{1}\right)$ can be associated with $\phi(\boldsymbol{x}, t)>0$, while that of the second fluid $\left(\Omega_{2}\right)$, is defined by $\phi(\boldsymbol{x}, t) \leq 0$.

The conservation of $\phi$ in any control volume $V_{t} \subset \Omega$ which is moving with the divergence-free velocity field $\boldsymbol{u}$, can be defined by the following hyperbolic equation

$$
\left.\frac{\partial \phi}{\partial t}+(\boldsymbol{u} \cdot \nabla) \phi=0 \quad \text { in } \Omega, t \in\right] 0, t_{\mathrm{f}}[
$$

with the corresponding initial and boundary conditions:

$$
\begin{aligned}
& \left.\phi=\bar{\phi} \quad \text { on } \Gamma_{\mathrm{in}}, t \in\right] 0, t_{\mathrm{f}}[, \\
& \phi=\phi_{0} \quad \text { in } \Omega, t=0 .
\end{aligned}
$$

The initial condition $\phi_{0}$, is chosen in order to define the initial position of the interface, and at the same time provide a smooth field which can be easily advected. The boundary condition $\bar{\phi}$ determines an inflow boundary when it is needed. Usually an initial condition field $\phi_{0}$ such that $|\phi|=1$ is chosen. We use standard numerical techniques to approximate the level set equation (20), solving this equation at the end of each time step to define the interface between both fluids which is then used in the next time step. Due to its pure convective nature it requires some type of stabilization. In this work we use the classical SUPG technique for this purpose [42], which corresponds to a standard variational multiscale (VMS) one in a pure convection problem. Time is discretized using second order finite difference schemes (Crank-Nicolson in the examples). From time to time, re-distancing is needed to preserve the condition $|\phi|=1$, for example, when interfaces merge or by the advection nature of the equation (20), which we do using a direct calculation of the distance to $\phi=0$ of each node (see [18] for examples of alternatives).

\subsection{Discontinuous gradient pressure functions}

Using fixed meshes, the standard Lagrangian finite element approximation of an unknown $\psi$ within each element $K$ of the finite element partition can be written as:

$$
\left.\psi_{h}\right|_{K}=\sum_{I=1}^{n_{\text {nod }}} N_{K}^{I} \psi_{K}^{I}
$$



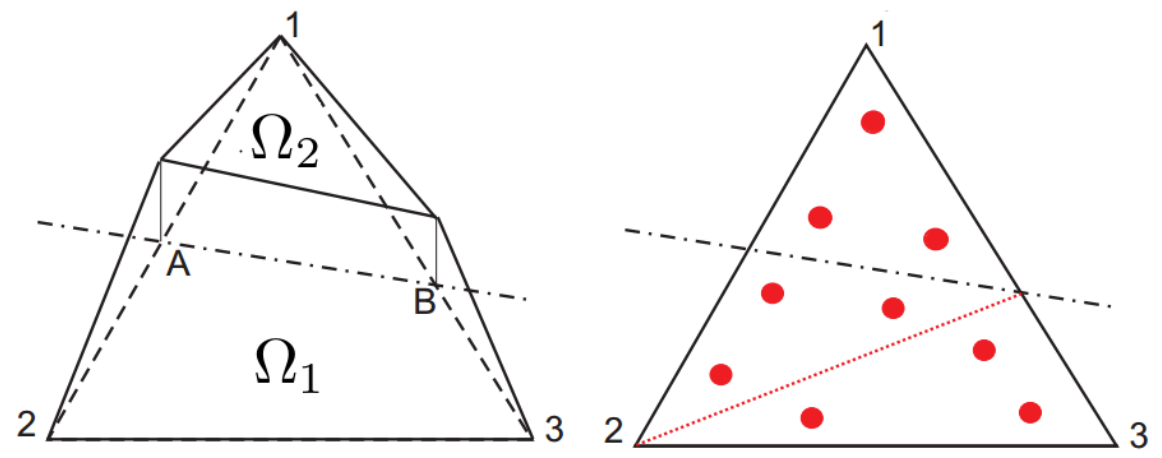

Figure 1: Enriched element sketch and modified integration rule.

where $n_{\text {nod }}$ is the number of element nodes, $N_{K}^{I}$ the shape function of node $I$ and $\psi_{K}^{I}$ the nodal value at this node. In typical finite element methods, the gradient of the shape functions is continuous within each element and therefore the gradient of the unknown is also continuous. If the interface cuts an element, the discontinuity in the material properties when solving a two immiscible fluid flow problems leads to discontinuities in the gradients of the unknowns which cannot be captured with standard finite element interpolation.

In this work we use the enriched gradient pressure method proposed in [14] to treat different density fluids. If an element is crossed by the interface, the pressure can be interpolated as

$$
\left.p_{h}\right|_{K}=\sum_{i=1}^{n_{\text {nod }}} N_{K}^{I} p_{K}^{I}+N_{K}^{*} p_{K}^{*}
$$

The enriched shape function $N_{K}^{*}$ has a constant gradient on each side of the interface, its value is zero at the element nodes and it is $C^{0}$ continuous in $K$. The added degree of freedom is local to the element and can therefore be condensed after the element matrix has been computed and before the assembly step. As a consequence, the number of nodes of the problem remains constant. It is worth noting that this is the main difference between our approach and that of a XFEM-strategy [13], in which the enriching function would be multiplied by the standard shape functions (making use of the fact that they are a partition of unity). This would introduce new pressure degrees of freedom at the nodes of the elements cut by the interface.

In our approach, the resulting pressure finite element space is defined by discontinuous functions across the inter-element boundaries, and thus it is a subspace of $L^{2}(\Omega)$, but not of $H^{1}(\Omega)$, as would be the case using $P 1-P 1$ elements. However, the method is still conforming [14].

Figure 1 (left) shows a sketch of the enriched pressure used for an element crossed by the interface in a $P 1$ element. In the same figure (right) the modified integration rule used to ensure a good representation of the discontinuities in the cut elements is shown. This modified integration rule was used in all numerical examples, independently of whether the enriched pressure functions were used or not. In the 2D case the element is subdivided into three sub-elements (for $P 1$ elements) while in the $3 \mathrm{D}$ case for linear elements it can be subdivided into four or six sub-elements (sub-tetrahedra), depending on the cut type. For each sub-element the same integration rule as for the original elements is used.

Let us consider linear triangles in $2 \mathrm{D}$ first. The enriched shape functions can be constructed using the 

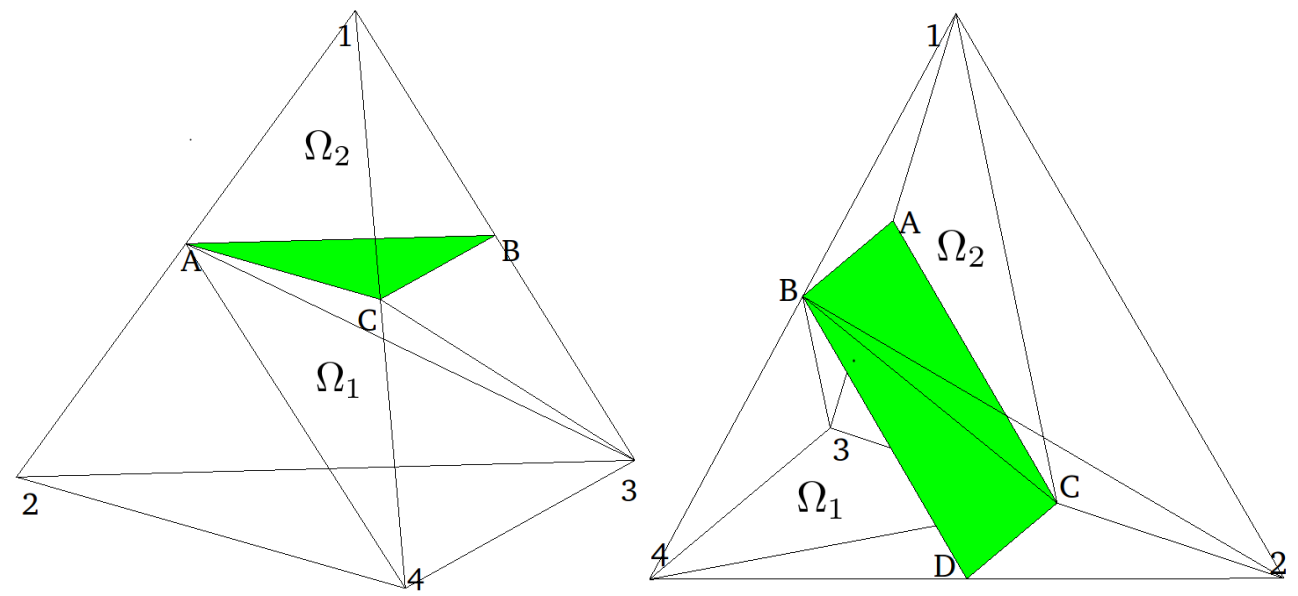

Figure 2: Tetrahedral element cut: three intersection points (left) and four intersection points (right).

standard shape functions and the position of the intersection points (A and B in Figure 1) as

$$
N_{K}^{*}=\left\{\begin{array}{c}
\theta_{1} N_{K}^{1}, \text { on } K_{1}:=\Omega_{1} \cap \Omega_{K}, \\
\theta_{2} N_{K}^{2}+\theta_{3} N_{K}^{3}, \text { on } K_{2}:=\Omega_{2} \cap \Omega_{K}
\end{array}\right.
$$

were $\theta_{i}, i=1,2,3$, are parameters to be determined. To construct each component of the enriched function we can follow the next three steps:

- Assume that $N_{K}^{*}\left(\boldsymbol{x}_{A}\right)=1$ (the other case $N_{K}^{*}\left(\boldsymbol{x}_{B}\right)=1$ is also possible).

- Use this definition to evaluate each component of the enriched function, i.e., $\left.N_{K}^{*}\left(\boldsymbol{x}_{A}\right)\right|_{K_{1}}=\left.N_{K}^{*}\left(\boldsymbol{x}_{A}\right)\right|_{K_{2}}=$ 1, and the fact that $N_{K}^{3}\left(\boldsymbol{x}_{A}\right)=0$.

- The enriched function must be continuous in $\Omega_{K}$, so $\left.N_{K}^{*}\left(\boldsymbol{x}_{B}\right)\right|_{K_{1}}=\left.N_{K}^{*}\left(\boldsymbol{x}_{B}\right)\right|_{K_{2}}$. In the same way as in the above step, $N_{K}^{2}\left(\boldsymbol{x}_{B}\right)=0$.

Using the above three points and based on the cut element showed in Figure 1, the parameters $\theta_{i}, i=$ $1,2,3$, are:

$$
\theta_{1}=\frac{1}{N_{K}^{1}\left(\boldsymbol{x}_{A}\right)}, \theta_{2}=\frac{1}{N_{K}^{2}\left(\boldsymbol{x}_{A}\right)}, \theta_{3}=\theta_{1} \frac{N_{K}^{1}\left(\boldsymbol{x}_{B}\right)}{N_{K}^{3}\left(\boldsymbol{x}_{B}\right)} .
$$

The same ideas can be used to obtain $N_{K}^{*}$ for $3 \mathrm{D}$ linear elements or for other element types. In the tetrahedral case we have two possibilities, depending on the number of intersection points (3 or 4 ) as we can see in figure 2, where the intersection cut plane is defined by the green area.

For the three intersection points case, the enriched pressure function takes the following form:

$$
N_{K}^{*}=\left\{\begin{array}{c}
\theta_{1} N_{K}^{1}, \text { on } K_{1}=\Omega_{1} \cap \Omega_{K}, \\
\theta_{2} N_{K}^{2}+\theta_{3} N_{K}^{3}+\theta_{4} N_{K}^{4}, \text { on } K_{2}=\Omega_{2} \cap \Omega_{K}
\end{array}\right.
$$

where

$$
\theta_{1}=\frac{1}{N_{K}^{1}\left(\boldsymbol{x}_{A}\right)}, \theta_{2}=\frac{1}{N_{K}^{2}\left(\boldsymbol{x}_{A}\right)}, \theta_{3}=\theta_{1} \frac{N_{K}^{1}\left(\boldsymbol{x}_{B}\right)}{N_{K}^{3}\left(\boldsymbol{x}_{B}\right)}, \theta_{4}=\theta_{1} \frac{N_{K}^{1}\left(\boldsymbol{x}_{C}\right)}{N_{K}^{4}\left(\boldsymbol{x}_{C}\right)}
$$




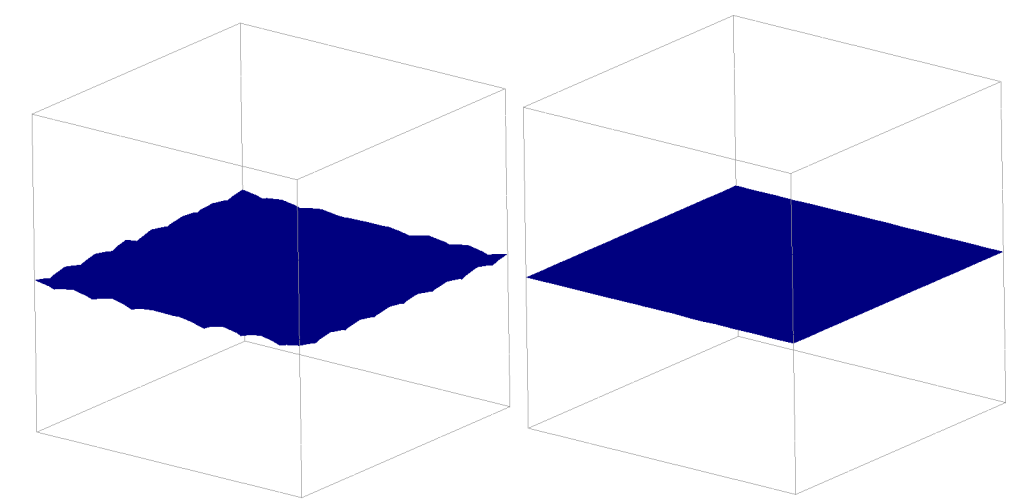

Figure 3: Three-dimensional hydrostatic pressure problem: without enriched functions (left) and using enriched functions (right).

In the case of four intersection points we have:

$$
N_{K}^{*}=\left\{\begin{array}{c}
\theta_{1} N_{K}^{1}+\theta_{2} N_{K}^{2}, \text { on } K_{1}=\Omega_{1} \cap \Omega_{K}, \\
\theta_{3} N_{K}^{3}+\theta_{4} N_{K}^{4}, \text { on } K_{2}=\Omega_{2} \cap \Omega_{K}
\end{array}\right.
$$

with

$$
\theta_{1}=\frac{1}{N_{K}^{1}\left(\boldsymbol{x}_{A}\right)}, \theta_{2}=\theta_{3} \frac{N_{K}^{3}\left(\boldsymbol{x}_{C}\right)}{N_{K}^{2}\left(\boldsymbol{x}_{C}\right)}, \theta_{3}=\frac{1}{N_{K}^{3}\left(\boldsymbol{x}_{A}\right)}, \theta_{4}=\theta_{2} \frac{N_{K}^{2}\left(\boldsymbol{x}_{D}\right)}{N_{K}^{4}\left(\boldsymbol{x}_{D}\right)} .
$$

From the numerical point of view, when the cut is extremely near to a nodal point, bad or poor conditioning of the additional degrees of freedom can occur, causing the new shape functions to have large gradients. In order to minimize this problem, an external numerical parameter can be defined to "move" the fluid-interface over the node, for example, if the distance between the geometric center of the intersection surface (line in $2 \mathrm{~d}$ ) to a nodal point is smaller than $\epsilon=0.02 h$ (where $h$ is the element diameter). In this case, the interface is moved over the node. This numerical treatment is sufficient to solve ill-conditioning problems when dealing with free surface. However, the ill-conditioning present in the two-fluid flow problem is independent of the cut criteria and is truly associated to the ratio between the properties of the fluids. The larger this ratio is, the more ill-conditioned the final problem will be. No remedies for this type of ill-conditioning can be applied, and the linear system solver preconditioner will be in charge of improving the condition number of the system to be solved.

The most illustrative example where the need of some kind of enrichment is crucial is in fact, not a flow, but two different density fluids at rest (the lighter one on top) inside a closed cavity. The hydrostatic pressure gradient is discontinuous at the interface, and therefore this cannot be correctly represented by the standard finite element shape functions. Since the problem is coupled in velocities, stresses and pressure, the error in the representation of the pressure gives rise to spurious velocities that in turn give rise to spurious stresses. Figure 3 shows the interface solution of a two-fluid flow problem using a Giesekus fluid and a Newtonian fluid on top. The Newtonian fluid is one hundred times lighter than the Giesekus fluid in the bottom.

Remark: A fully enriched method for a viscoelastic two-fluid flow problem, should include enrichment for the three fields (velocity-stress-pressure). In this work we use the pressure enrichment as a first approach to show that the two-fluid flow problem cannot be solved accurately without enrichment. 

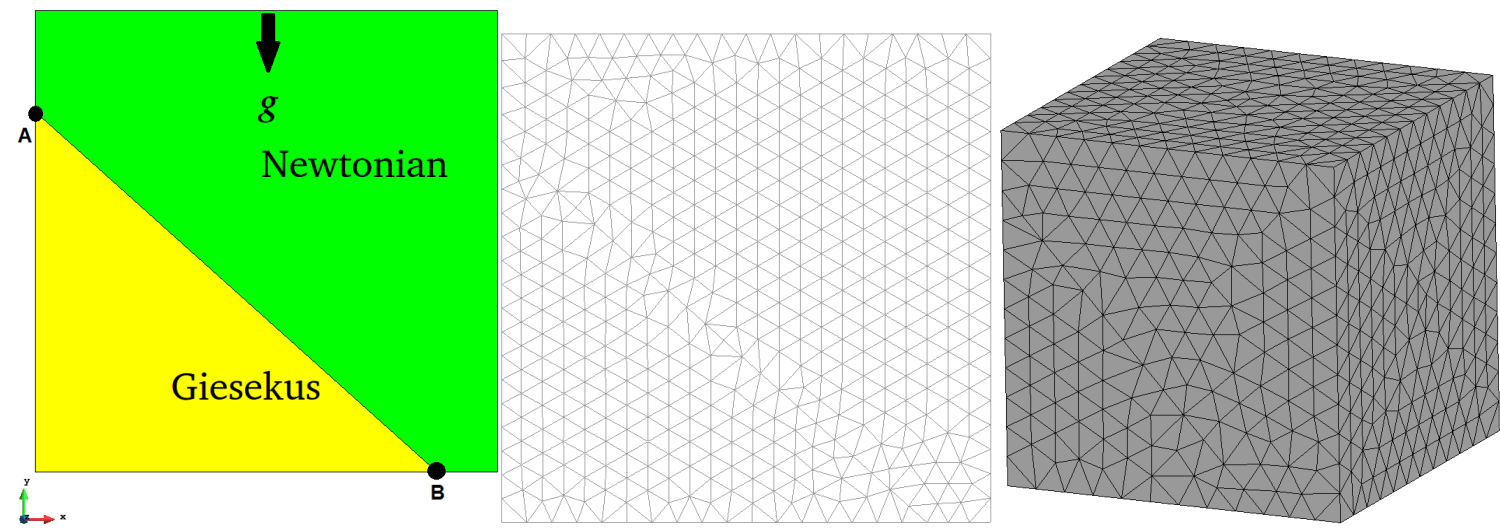

Figure 4: Two-fluids cavity problem: Initial condition, mesh used in 2D and mesh used in 3D (from left to right ,respectively).

\section{Numerical results}

In this section, some numerical tests are conducted to show the performance of the proposed methods. In subsection 5.1 a classical sloshing of a Giesekus fluid inside a covered square cavity with a one hundred times lighter Newtonian fluid on top is numerically modeled. Subsection 5.2 is devoted to the jet buckling problem of an Oldroyd-B fluid. In both examples we solve the two-dimensional and the three-dimensional cases.

In all the numerical examples the discrete and linearized problem is solved using an iterative solver based on the BiCGStab (Stabilized version of BiConjugate Gradient Squared) method of van der Vorst [43], with the additive Schwarz preconditioner.

\subsection{Sloshing of two fluids in a square covered cavity}

The sloshing problem is a typical benchmark to test the accuracy of new numerical methods widely used in Newtonian fluids and less explored in the non-Newtonian case [44, 45]. In this case we solve the sloshing of a Giesekus fluid inside a square covered cavity with a Newtonian fluid on top with the properties detailed in table 1. The initial condition is an unstable inclined plane, as shown in Figure 4, under the influence of gravitational forces. For the time discretization both in the two-dimensional and in the three-dimensional case we use the BDF2 scheme for the momentum and constitutive equations, and the Crank Nicolson scheme for the level set equation. The time step has been taken constant with a value $\delta t=0.01$ in all the cases.

\begin{tabular}{|c|c|c|c|c|c|}
\hline Fluid type & $\eta_{0, i}$ & $\beta_{i}$ & $\lambda_{i}$ & $\varepsilon_{i}$ & $\rho_{i}$ \\
\hline Giesekus & 1.0 & 0.111 & 1.0 & 0.01 & 100.0 \\
\hline Newtonian & 1.0 & 0.111 & 0.0 & 0.0 & 1.0 \\
\hline
\end{tabular}

Table 1: Fluid properties in the sloshing problem.

The space discretization used in the sloshing case is shown in Figure 4. The meshes used are very coarse, but enough for our purposes. For the 2D case, the mesh is defined by 890 linear elements, while in the threedimensional case 76742 linear tetrahedral elements are used. The coordinates of points A and B detailed in Figure 4 are $(0.78 ; 0)$ and $(0.87 ; 0)$ respectively.

The objective of this subsection is to show that a standard formulation cannot be used to solve accurately a two-fluid flow problem without the addition of some type of enrichment. The enrichment used here is added only to the pressure field, although the problem has different properties also in the "viscoelastic" parameters. 


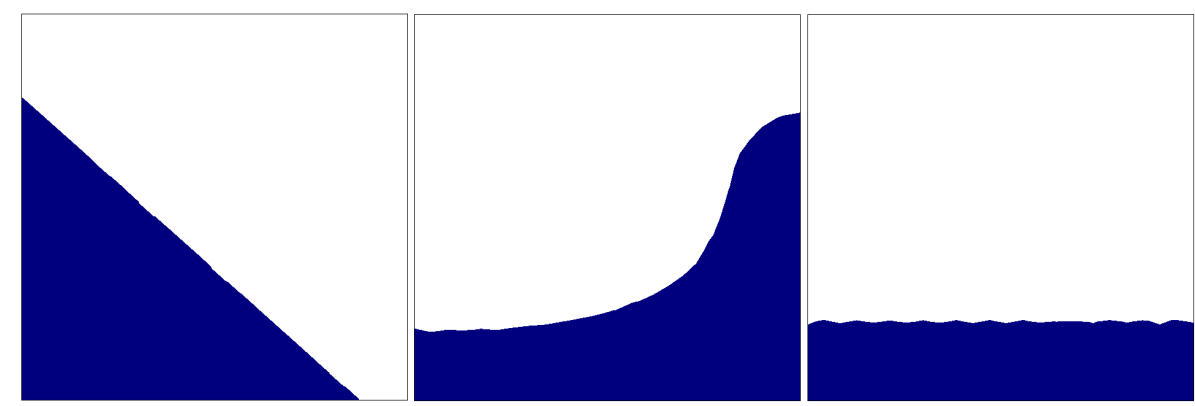

a)

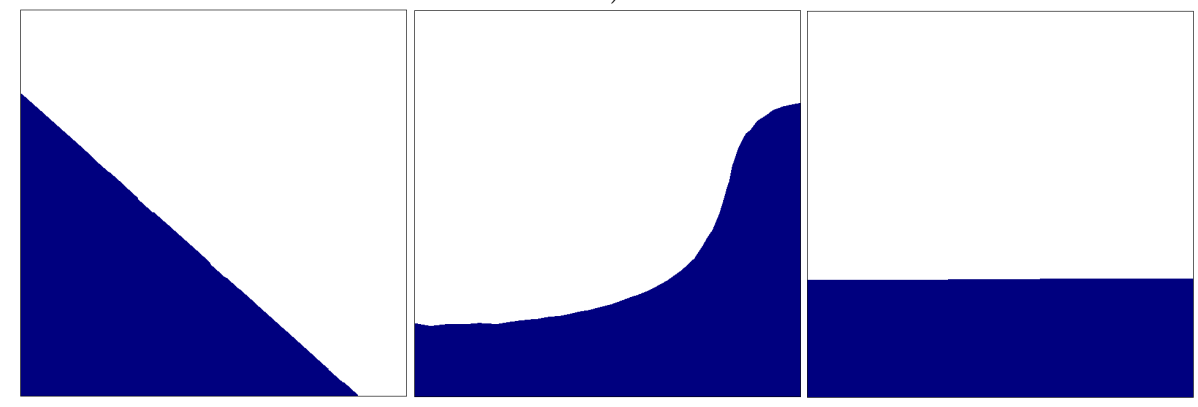

b)

Figure 5: two-dimensional sloshing: a) without enriched functions and b) using enriched functions $(t=0, t=0.7$ and $t=30$ for both cases from left to right).

The dimensionless numbers of the problem based on the maximum obtained velocity $\left(|\boldsymbol{u}|_{\max } \approx 3.5\right)$ and the characteristic length of the cavity $\left(L_{c}=1\right)$, are for the $2 \mathrm{D}$ case:

$$
\operatorname{Re} \approx 350, \text { We } \approx 3.5, \text { Fr } \approx 1.1
$$

and for the three-dimensional case based on the maximum velocity $\left(|\boldsymbol{u}|_{\max } \approx 4.5\right)$ :

$$
\operatorname{Re} \approx 450, \mathrm{We} \approx 4.5, \mathrm{Fr} \approx 1.43
$$

where Re represents the Reynolds number, We the Weissenberg number and Fr the Froude number. Note that the velocity decreases as the flow becomes stationary, and so do these numbers.

In Figure 5 we have the results obtained with and without enriched pressure functions for the 2D case. The most important point of these results is the final state of both cases. Without pressure enrichment, in addition to a greater amount of mass losses, the state of rest, where the interface must be completely horizontal is not satisfied. In the case of enriched pressure the lost mass is around $9 \%$, while in the nonenriched case a mass loss of $38.5 \%$ is obtained. It is important to note that the mesh used is coarse and the time step large, but we think that this is the best scenario to compare both methods.

The inclusion of a discontinuity-capturing term is necessary according to our experience in the flow of viscoelastic fluids. In Figure 6 the temporal history of the bottom right corner of the stress magnitude in the time interval $[0,3]$ is shown, with and without a discontinuity-capturing term for the two-dimensional case of the sloshing problem. It is clear from this figure that a discontinuity-capturing term can help to eliminate possible non-physical peaks. For all the cases shown in this work we use the discontinuity-capturing term 


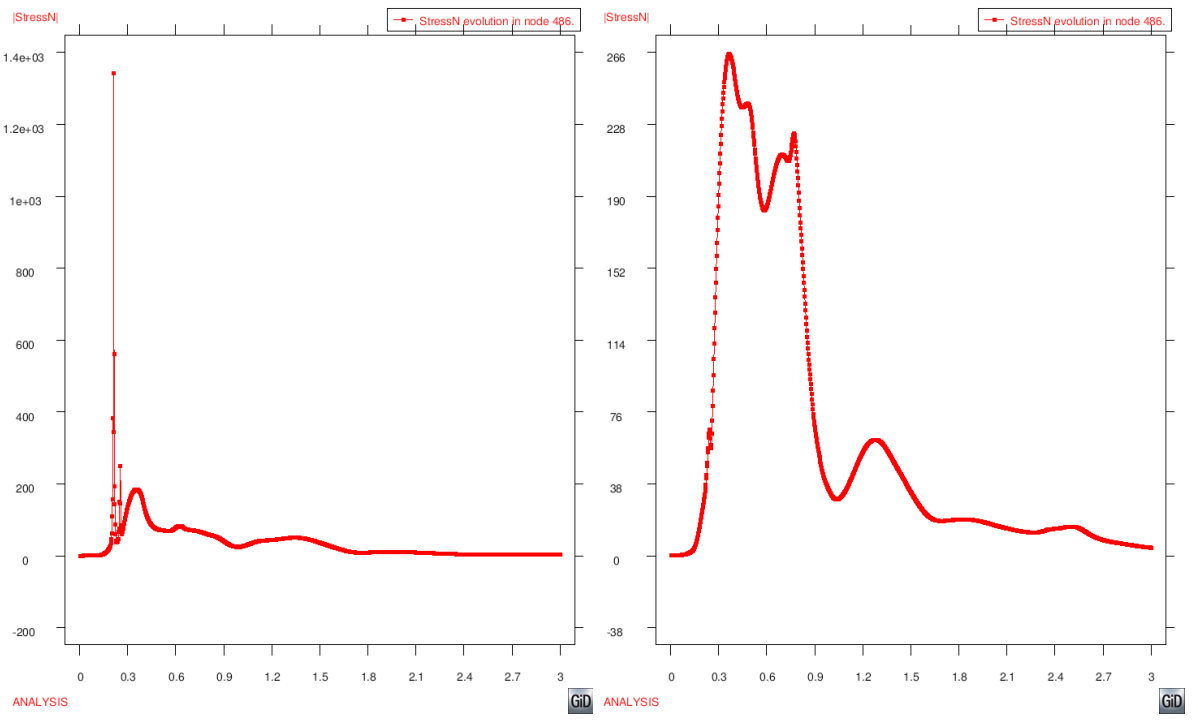

Figure 6: Temporal history of the bottom right corner of the stress magnitude without (left) and with (right) a discontinuitycapturing term.

summarized in subsection 3.3.

For the three-dimensional case, the tendency is maintained with less mass loss in the enriched pressure case $(13 \% \mathrm{v} / \mathrm{s} 35 \%)$, and with the capability to solve the hydrostatic steady state. The same that in the 2D case, respect to the mesh and time step size. In both cases the loss mass could be reduced upon mesh refinement or reducing the time step size.

In viscoelasticity the number of degrees of freedom per node can be a bottleneck from the computational point of view, especially in the three-dimensional case. In table 2 a comparison of total CPU time and the maximum memory required to solve the problem both in the two-dimensional and in the three-dimensional cases are shown. It can be observed that the use of a fractional step scheme is critical, especially in the threedimensional case. The CPU time is reduced by a factor of three and the maximum memory requirements by a half. Regarding the accuracy of the numerical solution, the results obtained by the fractional step approach are practically identical to those of the monolithic approach.

\begin{tabular}{|c|c|c|c|}
\hline & Monolithic & Fractional & Fractional/Monolithic \\
\hline \hline $2 \mathrm{D}-\mathrm{CPU}_{\text {time }}$ & $1828.1 \mathrm{~s}$ & $1267.14 \mathrm{~s}$ & 0.6931 \\
\hline $3 \mathrm{D}-\mathrm{CPU}_{\text {time }}$ & $158747 \mathrm{~s}$ & $61861 \mathrm{~s}$ & 0.3896 \\
\hline $2 \mathrm{D}-$ Memory & $16.7 \mathrm{Mb}$ & $14.04 \mathrm{Mb}$ & 0.84 \\
\hline $3 \mathrm{D}-$ Memory & $547 \mathrm{Mb}$ & $301 \mathrm{Mb}$ & 0.55 \\
\hline
\end{tabular}

Table 2: Comparison of computational requirements: monolithic approach $\mathrm{v} / \mathrm{s}$ fractional step approach.

\subsection{Jet buckling problem}

The jet buckling problem is the typical benchmark of free surface problems in viscoelastic fluids. In this subsection we use the two-fluid flow approach with enriched pressure shape functions to solve it. The buckling phenomena of viscous flows is a known physical instability, that depends in Newtonian fluids on the Reynolds number and the height from which the jet falls. Tomé and McKee [46] give a complete analysis of planar jets 


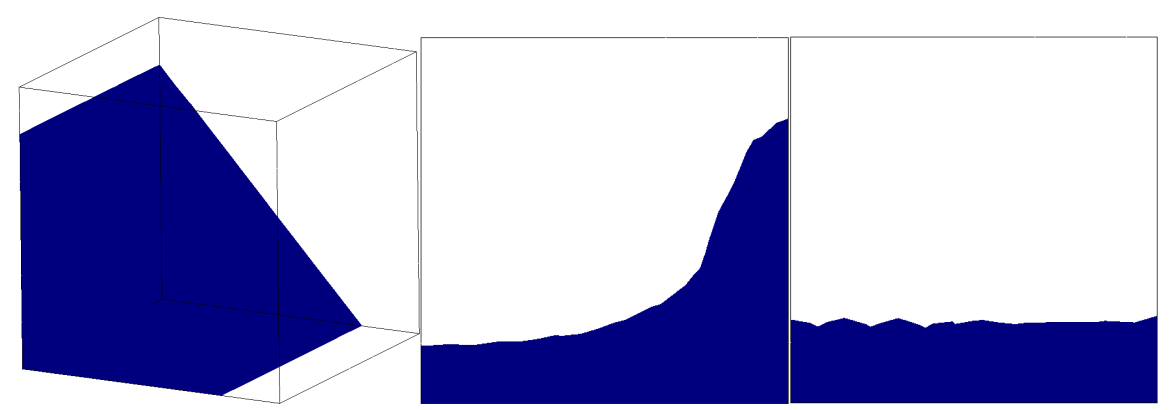

a)

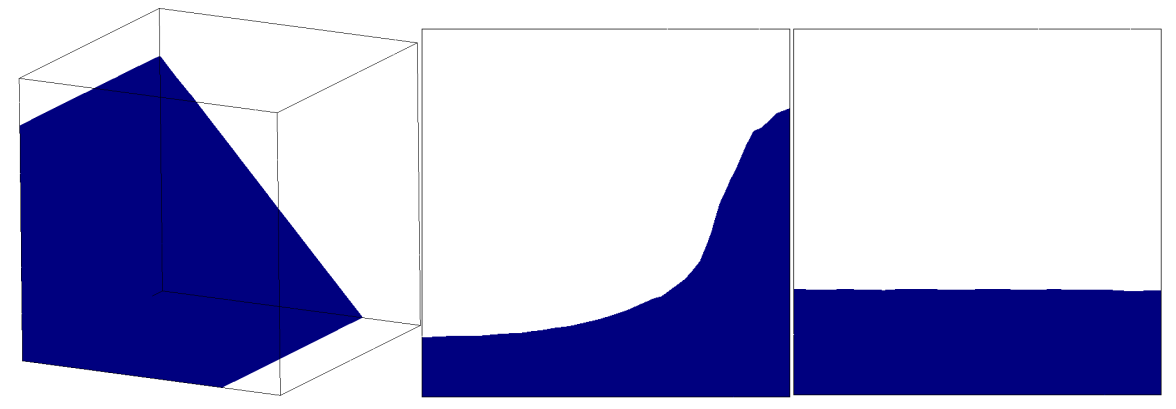

b)

Figure 7: Three-dimensional sloshing: a) without enriched functions and b) using enriched functions $(t=0, t=0.7$ and $t=15$ in both cases from left to right).

in Newtonian fluids. The authors propose an empirical relationship to evaluate the critical Reynolds number that defines the beginning of buckling which depends on the characteristic lengths that define the problem in the two-dimensional case. Another important work in viscous buckling analysis from the experimental and theoretical points of view is due to Cruickshank and Munson [47], where the authors show that a necessary condition for a jet buckling is Re $\leq 1.2$. In the work of Bonito et al. [5] the authors use the relationship of Tomé and McKee to compare the flow patterns in viscoelastic and Newtonian fluids.

In Figure 8 the problem definition and the meshes used are shown for the two-dimensional and the threedimensional cases. For rigid boundaries we employ slip walls (no-slip walls is another used option). The aspect ratios selected for the planar jet are $W / D=10$ and $H / D=20$ to ensure the occurrence of buckling (according to [46] buckling occurs for $H / D>8.8$ in Newtonian fluids). The fluid properties are defined with respect to the Reynolds number $(\mathrm{Re})$, the Weissenberg number (We), the inflow diameter $\left(D_{H}\right)$ and the inflow velocity $\left(\boldsymbol{u}_{\text {in }}\right)$ in Table 3 .

\begin{tabular}{|c|c|c|c|c|c|}
\hline$D_{H}$ & $\boldsymbol{u}_{\text {in }}$ & $\rho$ & $\beta$ & $\eta_{0}$ & $\lambda$ \\
\hline \hline 0.05 & 1.0 & 1000.0 & 0.111 & $\frac{\operatorname{Re}}{\rho\left|\boldsymbol{u}_{\text {in }}\right| D_{H}}$ & $\frac{W e D_{H}}{\left|\boldsymbol{u}_{\text {in }}\right|}$ \\
\hline
\end{tabular}

Table 3: Fluid properties for the buckling problem.

Figure 8 shows a sketch of the jet buckling problem and the meshes used for the two-dimensional and the three-dimensional cases. The 2D mesh is composed by 19209 linear elements and the three-dimensional case by 544548 linear tetrahedral elements. The buckling problem is by definition non-symmetric; therefore, the three-dimensional case must be solved for the full domain. It is important to note that we solve a two incompressible fluid flow problem and then, outlets are needed to ensure the mass conservation. For the time 

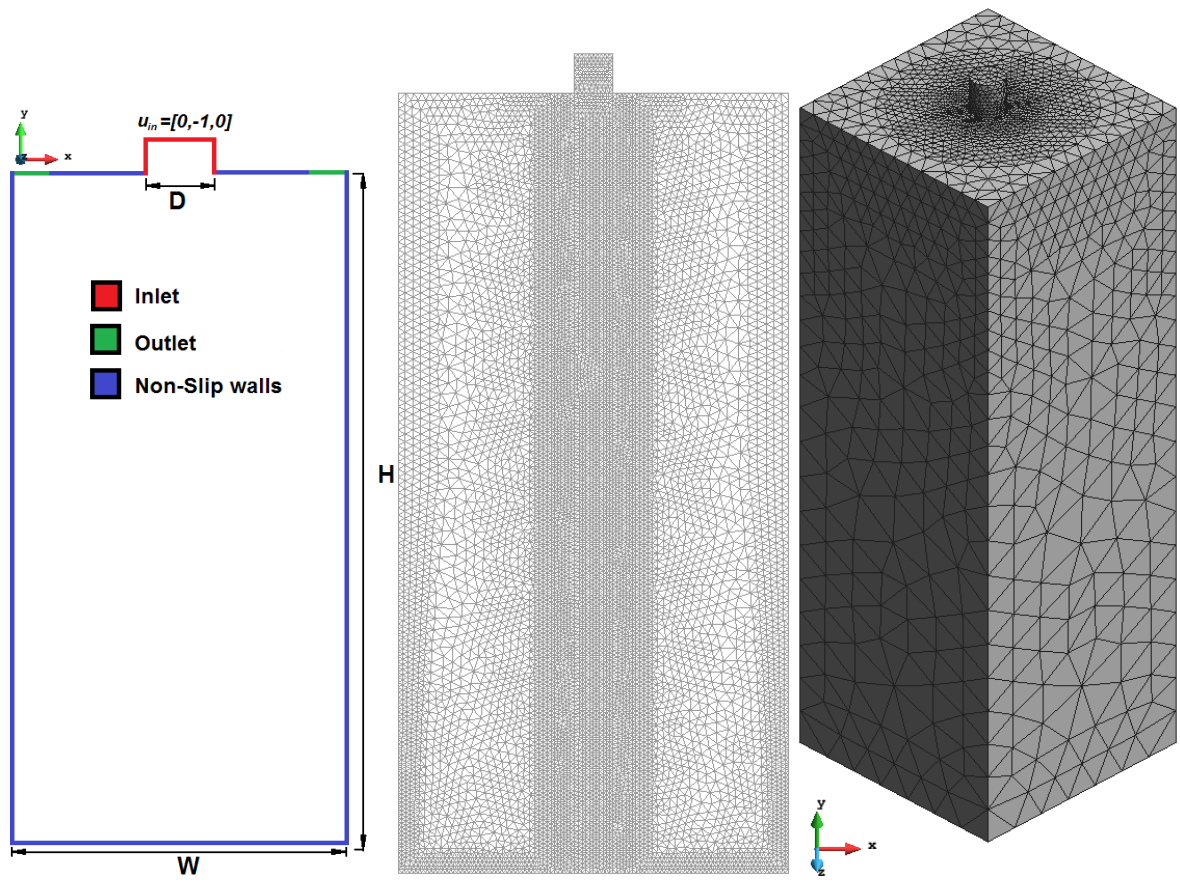

Figure 8: Jet buckling problem and mesh used.

discretization both in the two-dimensional and in the three-dimensional cases we use the BDF2 scheme for the momentum and the constitutive equations, and the BDF1 scheme for the level set equation. The time step was taken constant with a value $\delta t=0.005$ in all cases.

In general, the jet buckling problem is solved as a free surface case, i.e., the external flow is not solved. In this work we use the two-fluid flow approach (the real physical problem) to emulate the free surface-approach used in the literature. To do this, we use a ratio relationship between the densities and the viscosities, of the order of a water-air interaction problem. The values used for all the simulations are $\rho_{\text {jet }}=1000 \rho_{\text {external }}$ and $\eta_{0, \text { jet }}=500 \eta_{0 \text {,external. }}$. Obviously, in a two-fluid problem, the outer fluid affects the dynamics of the internal fluid. In our simulations we found that a density ratio $\rho_{\text {jet }} \geqslant 1000 \rho_{\text {external }}$ and a viscosity ratio $\eta_{0, \text { jet }} \geqslant 250 \eta_{0 \text {,external }}$, can be associated to a free-surface case. The real profit of a two fluid formulation is the capability to solve problems where the influence of the external flow is important, and therefore needs to be solved, which is not possible using a free surface approach.

With respect to mesh convergence, an analysis to ensure that the results are independent of the discretization used to perform the simulation is shown in Table 4. In this table, the the exact time for the impact of different fluids in the case $\mathrm{Re}=0.25$ is shown. The differences between mesh M2 and mesh M3 are negligible and, therefore, the selected mesh for all the calculations was mesh M2. In Table $4, t^{*}$ represents the instant of impact, $\mathrm{E}_{\%}$ the percentual error using as reference the values obtained using mesh M3 and $n_{e}$ the number of elements of the respective mesh. In this results, we can see how the shear-thinning effect of the fluid causes the impact to happen earlier than in the Newtonian case.

The first set of results is devoted to the validation of our formulation in the jet buckling for Newtonian 


\begin{tabular}{|c|c|c|c|c|}
\hline case & M1 $\left(\mathrm{n}_{\mathrm{e}}=6463\right)$ & $\mathrm{M} 2\left(\mathrm{n}_{\mathrm{e}}=19209\right)$ & $\mathrm{M} 3\left(\mathrm{n}_{\mathrm{e}}=58683\right)$ & \\
$\mathrm{Re}=0.25$ & $\Delta x_{\min }=0.0075$ & $\Delta x_{\min }=0.005$ & $\Delta x_{\min }=0.00333$ & \\
\hline & $t^{*} / \mathrm{E}_{\%}$ & $t^{*}$ & $t^{*} / \mathrm{E}_{\%}$ & \\
\hline $\mathrm{We}=0.0$ & $0.725 / 3.33 \%$ & $0.745 / 0.666 \%$ & 0.75 & \\
\hline $\mathrm{We}=10.0$ & $0.635 / 3.05 \%$ & $0.65 / 0.763 \%$ & 0.655 & \\
\hline $\mathrm{We}=100.0$ & $0.615 / 3.14 \%$ & $0.63 / 0.787 \%$ & 0.635 & \\
\hline
\end{tabular}

Table 4: Mesh convergence analysis in jet-buckling problem.

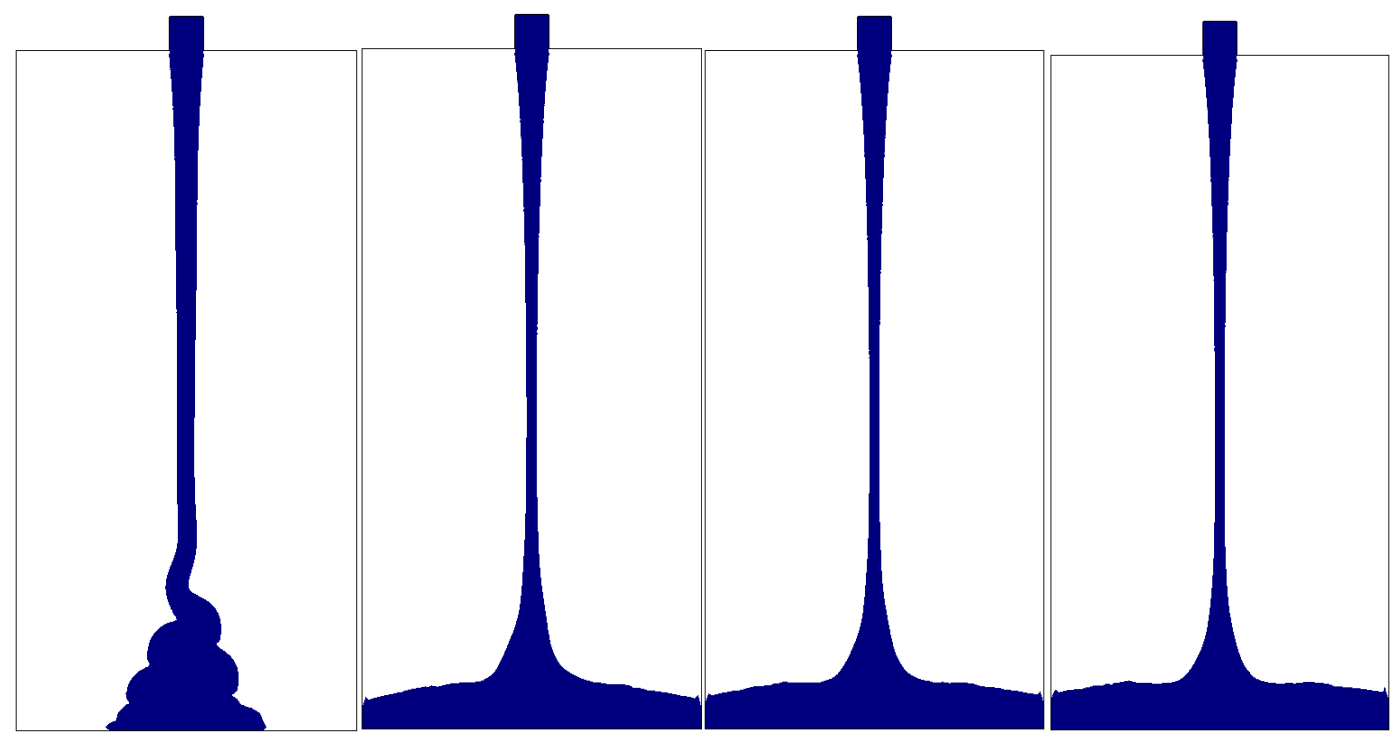

Figure 9: Jet buckling in Newtonian fluids. From left to right: $\operatorname{Re}=0.25, \operatorname{Re}=0.50, \operatorname{Re}=0.55$ and $\operatorname{Re}=0.60$ (all at $t=1.5$ )

fluids. From [46], the condition for a Newtonian jet to buckle is:

$$
\operatorname{Re}\left(\frac{H}{D}\right) \leq \sqrt{\frac{1}{\pi} \frac{\left(\frac{H}{D}\right)^{2.6}-8.8^{2.6}}{\left(\frac{H}{D}\right)^{2.6}}}
$$

where the Reynolds number is defined in terms of the inlet velocity and the total viscosity (i.e. $\operatorname{Re}=$ $\left.\rho\left|\boldsymbol{u}_{\text {in }}\right| D_{H} \eta_{0}^{-1}\right)$. For the ratio $H / D=20$, the critical Reynolds number for buckling is $\operatorname{Re} \approx 0.53$. The critical Reynolds number obtained in our work for Newtonian fluids is $\operatorname{Re} \approx 0.55$; for Re $\geqslant 0.6$ no sign of buckling exists (see Figure 9).

In Figure 10 the interface evolution of three cases was solved for $\operatorname{Re}=0.25$. This Reynolds number was selected to show the differences between Newtonian and viscoelastic fluid flows and the influence of the elastic properties in the buckling phenomena. The first row represents the Newtonian fluid while the second and third row are Oldroyd-B fluids defined by $\mathrm{We}=10$ and $\mathrm{We}=100$, respectively. In the first column, $t=0.6$, we can see as the elastic part of the fluid convects the flow, even in a fixed Reynolds number, advancing the instant of the impact point. For this Reynolds number case, the Newtonian fluid presents the classical buckling flow while the more elastic flow is completely different, showing that the fluid has an elastic resistance that prevents buckling at the beginning, generating an unstable fluid pile $(t=0.8$ and $t=0.9)$ that collapses later as we can see in Figure 10. Similar results were reported by Bonito et al. in [5] for the 
same case.

In Figures 11 and 12 we can see the contour lines for the normal stresses and for the pressure for the same steps shown in Figure 10 for both viscoelastic cases. The normal component $\sigma_{x x}$ reaches its maximum value when the flow strikes the ground and tries to slip. In this moment, the elastic properties of the fluid prevent slippage and the fluid begins to pile up. Later, the pile of fluid collapses and the other normal component of the stress tensor $\sigma_{y y}$ reaches its highest value. The pressure field reaches its highest value when the fluid hits the ground. In all the figures we can see the interface between both fluids clearly defined by a zone of high gradients characterized by a large concentration of contour lines.

For the three-dimensional case, the two limit cases used in the two-dimensional case were solved. For the Newtonian fluid case (Figure 13), the buckling occurs from the beginning in the same way as in the two-dimensional case. For the viscoelastic case (Figure 14), the flow hits the base in conditions similar to a more inertial flow, contrary to what is observed in the the Newtonian case $(t=1.0)$. Then, the elastic stresses become apparent by stopping the slipping of the jet and preventing buckling, and beginning the stacking of the fluid $(t=1.25, t=1.5)$. Finally, the stack begins to collapse $(t=2.0)$. Similar results were published by Bonito et al. in [5] using a free surface approach.

The resolution of a two-fluid flow problem gives the possibility to evaluate the interaction of both flows. Figure 15 gives a good graphical visualization of this point. This figure corresponds to the viscoelastic case where the outlets and the vortex zone near the jet are clearly defined.

The viscoelastic fluid flow problem is defined by a nonlinear system of equations, which is linearized and solved iteratively to reach an accepted convergence error value $\left(10^{-6}\right.$ in our simulations $)$ defined as

$$
E_{C V}^{i+1}=\frac{\left|\mathbf{U}^{i+1}-\mathbf{U}^{i}\right|}{\left|\mathbf{U}^{i+1}\right|}
$$

where $i$ represents the iteration counter. For the pressure and the stresses, the error is defined in the same way.

In each iteration the BiCGStab method is used to solve the system of equations. The Weissenberg number is the non-dimensional number that defines the non-linear nature of the fluid. In Table 5, the mean number of non-linear iterations required in each of the simulations is detailed and represented by $\overline{\mathrm{n}}_{i t}$. In this table we can see how the number of nonlinear iterations increases when the elastic behavior becomes dominant.

\begin{tabular}{|c|c|c|}
\hline $\mathrm{Re}=0.25$ & 2D-Case & 3D-Case \\
\hline \hline & $\overline{\mathrm{nn}}_{i t}$ & $\overline{\mathrm{n}}_{i t}$ \\
\hline $\mathrm{We}=0.0$ & 9 & 8 \\
\hline $\mathrm{We}=10$ & 18 & - \\
\hline $\mathrm{We}=100$ & 39 & 37 \\
\hline
\end{tabular}

Table 5: Non-linear iterations for the jet buckling-problem.

\section{Conclusions}

In this paper we have presented a stabilized finite element method to solve the two immiscible fluid flow problem by the level set method for Oldroyd-B and Giesekus fluids. The method shows very good stability and robustness even using the standard formulation of the elastic stress tensor. The buckling problem was 


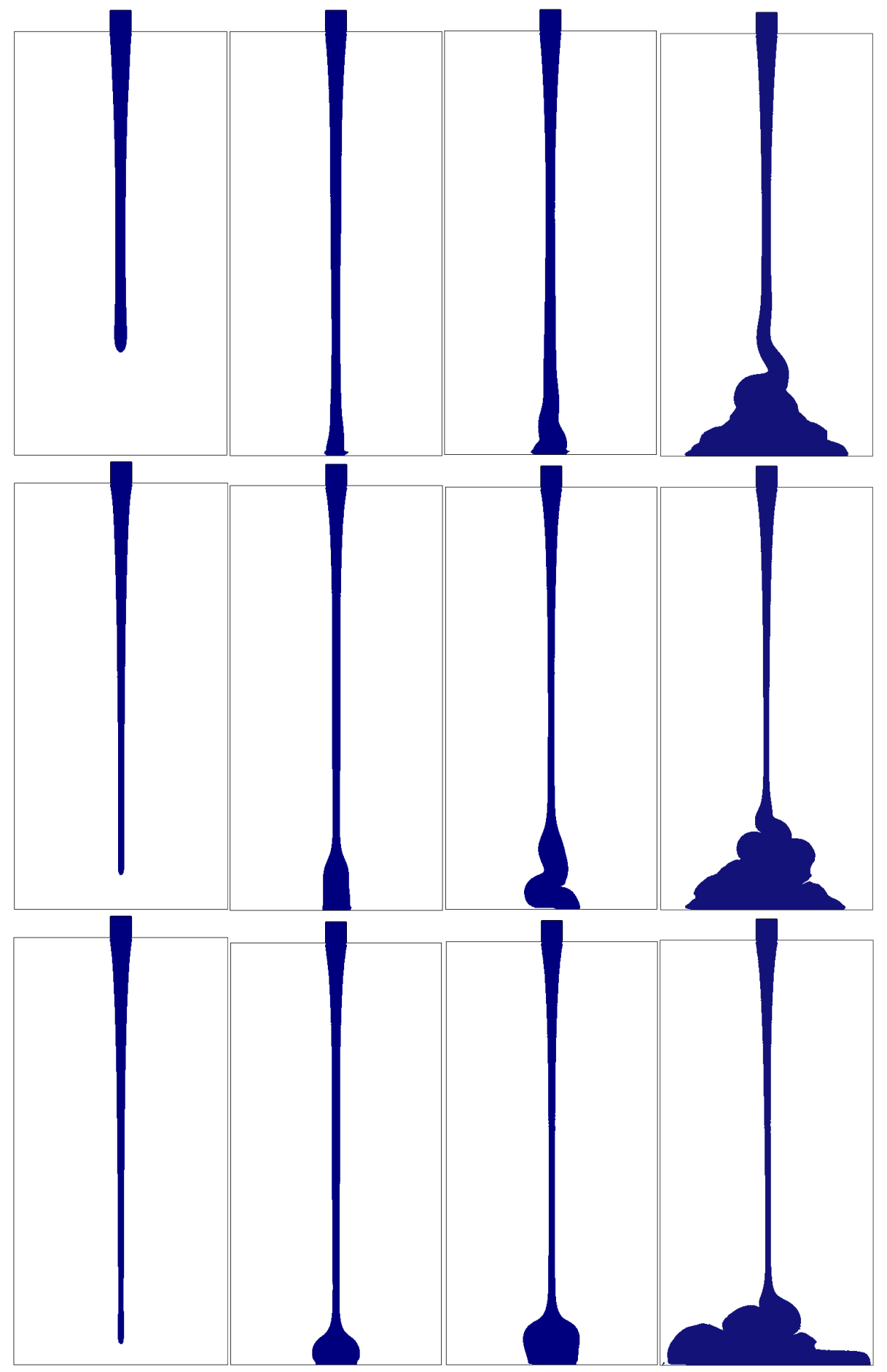

Figure 10: Jet buckling in a $2 \mathrm{D}$ rectangular cavity $(\mathrm{Re}=0.25)$. First row $\mathrm{We}=0$, second row $\mathrm{We}=10$ and third row We $=100$, at time $t=0.6$ (first column), $t=0.8$ (second column), $t=0.9$ (third column) and $t=1.5$ (fourth column). 


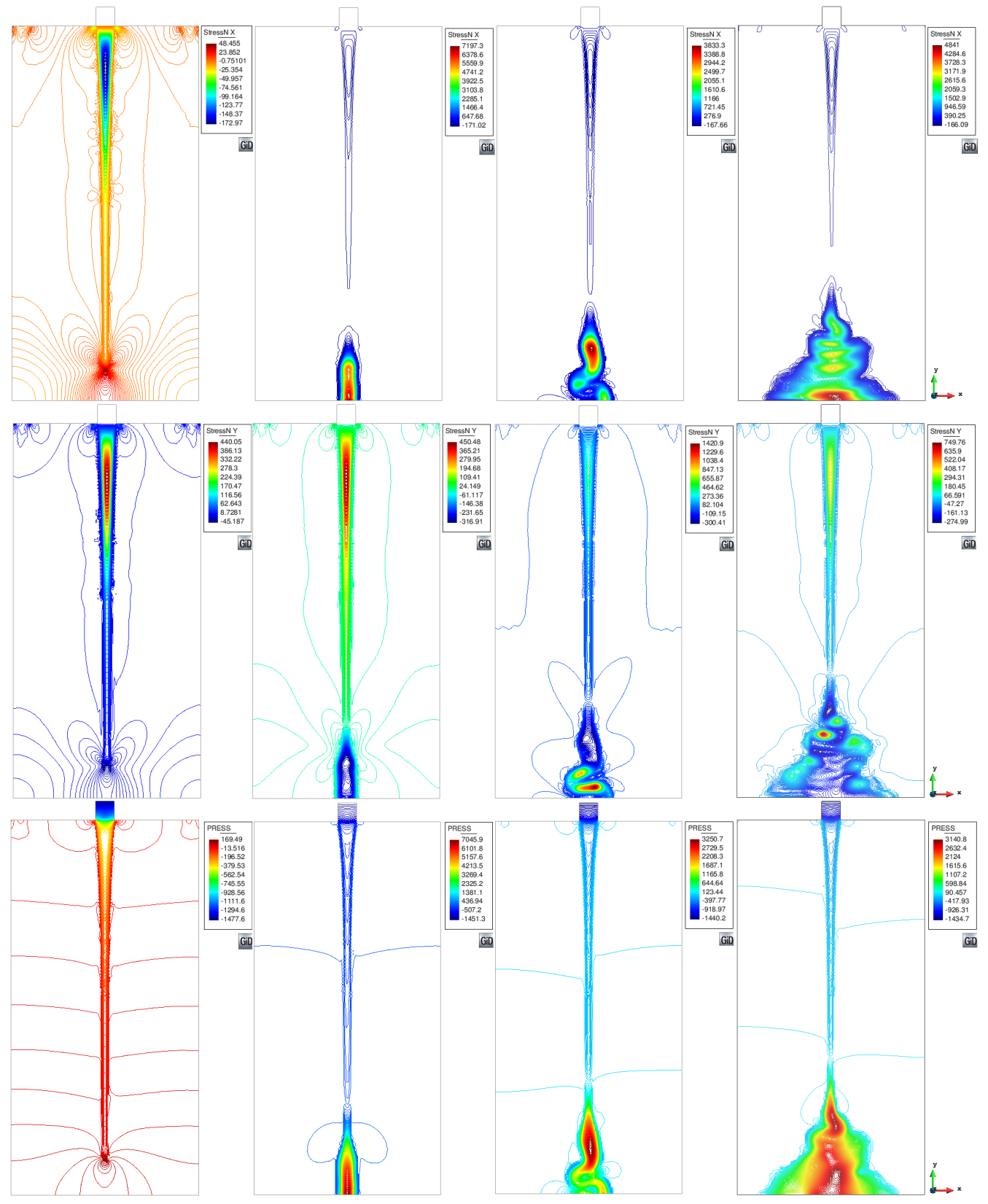

Figure 11: Contour line in the jet buckling problem for We $=10: \sigma_{x x}$ (first row), $\sigma_{y y}$ (second row) and $p$ (third row). For $t=0.6, t=0.8, t=0.9$ and $t=1.5$ (from left to right). 

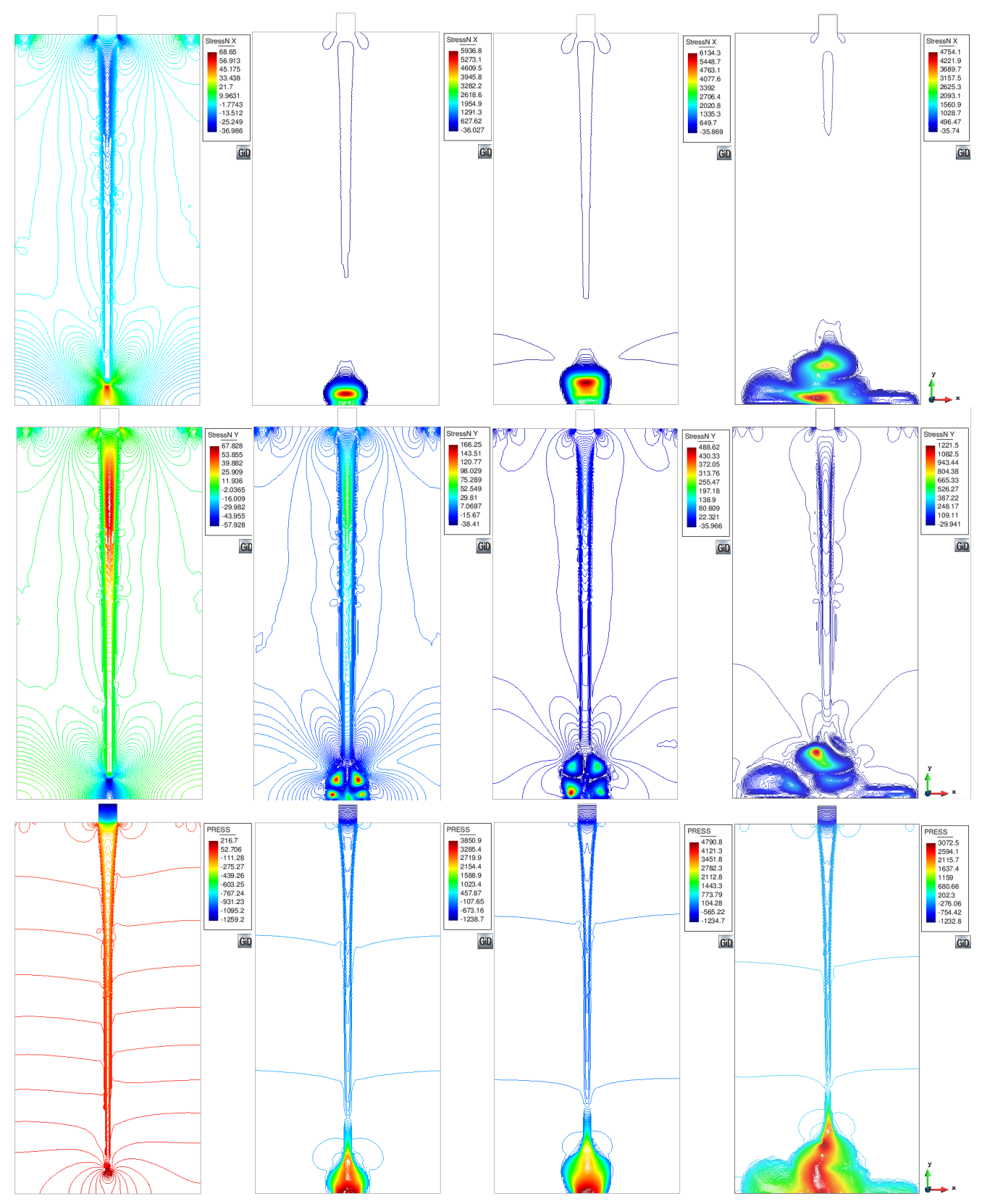

Figure 12: Contour line in the jet buckling problem for We $=100: \sigma_{x x}$ (first row), $\sigma_{y y}$ (second row) and $p$ (third row). For $t=0.6, t=0.8, t=0.9$ and $t=1.5$ (from left to right). 

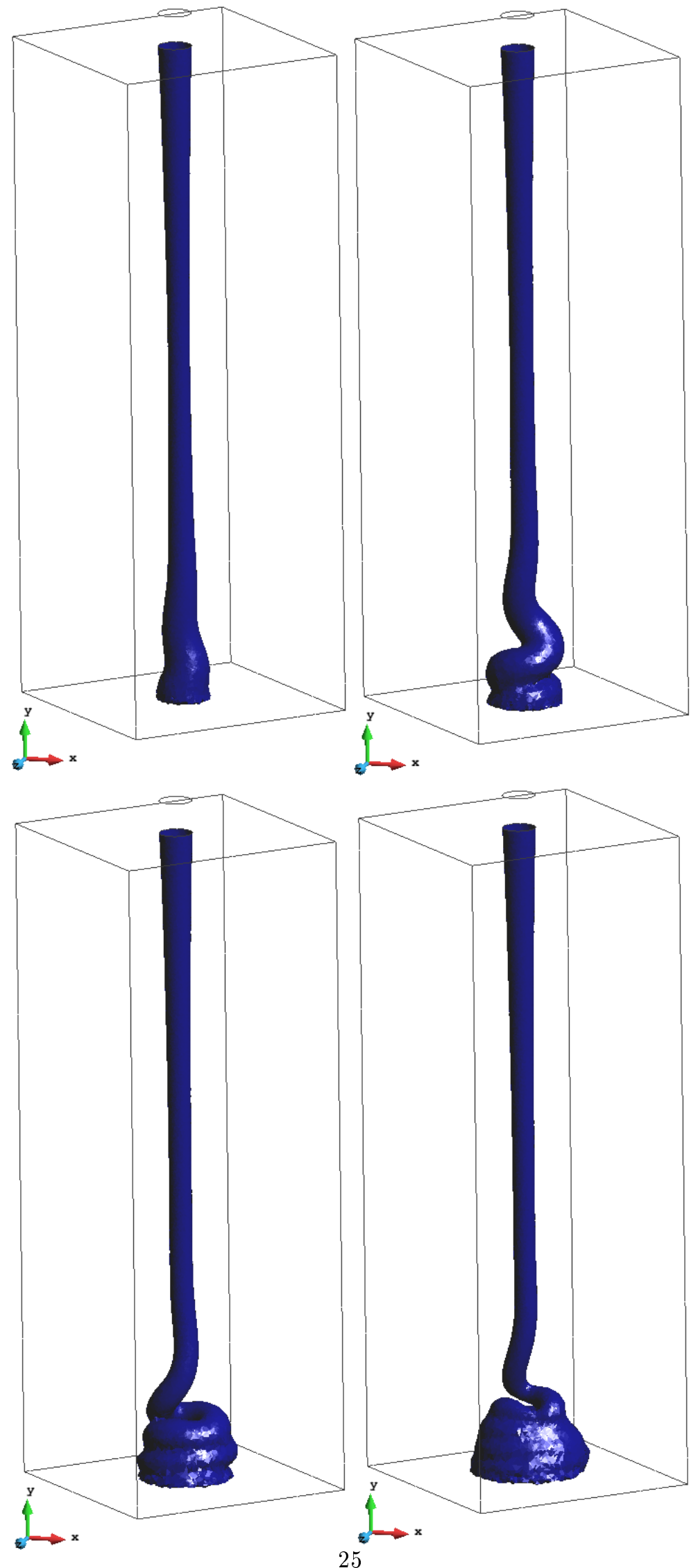

Figure 13: Jet buckling in a 3D prismatic cavity using a Newtonian fluid ( $\operatorname{Re}=0.25$, We $=0$ ). Top $(t=1.25$ and $t=1.5$ ), bottom $(t=1.75$ and $t=2)$. 

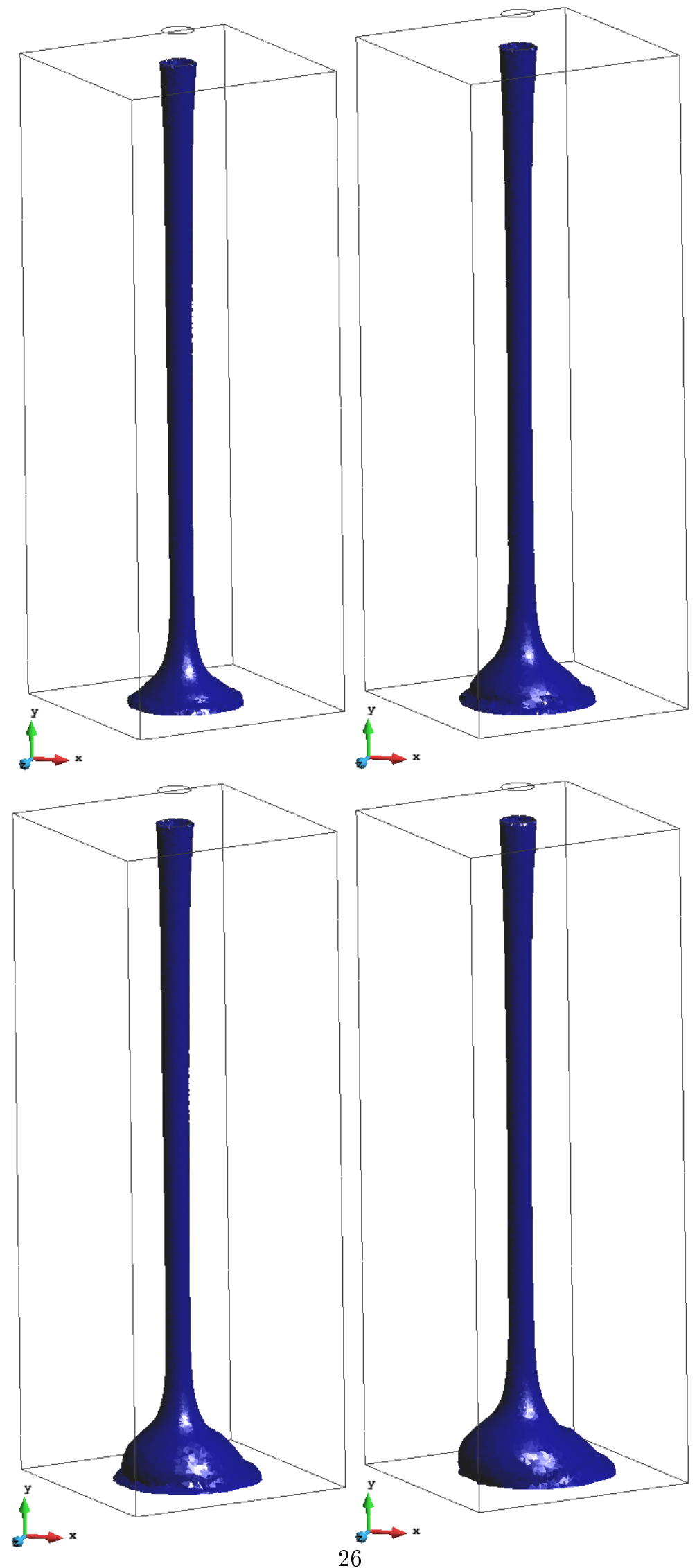

Figure 14: Jet buckling in a 3D prismatic cavity using a non-Newtonian $(\operatorname{Re}=0.25, \mathrm{We}=100)$. Top $(t=1.25$ and $t=1.5)$, bottom $(t=1.75$ and $t=2)$. 

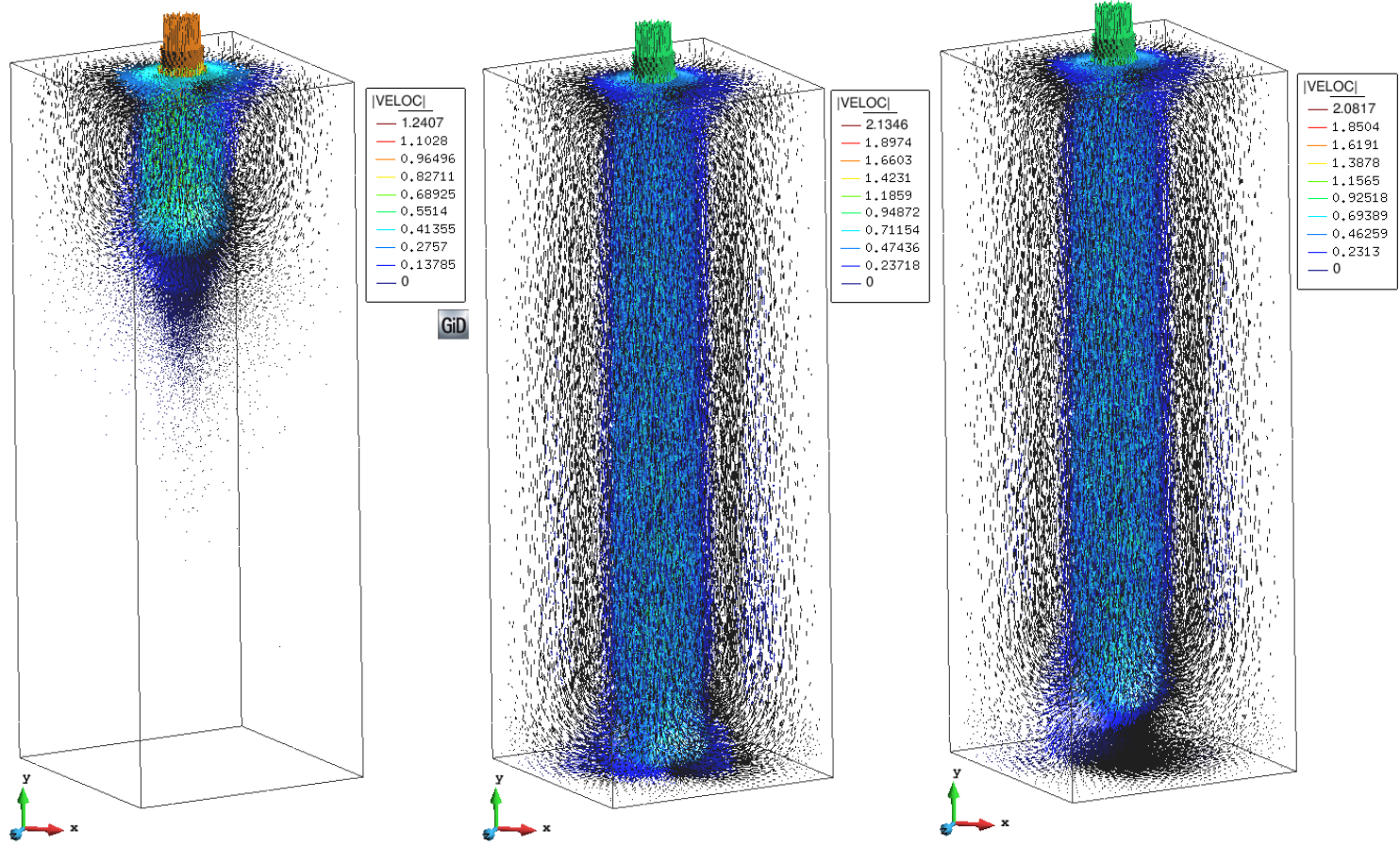

Figure 15: Velocity vectors in the jet buckling problem for We $=100$ in $t=0.2, t=1.0$ and $t=2.0$ (from left to right).

solved up to $\mathrm{We}=100$ without any sign of instability both in the two-dimensional and three-dimensional cases. The enriched pressure formulation permits the resolution even of "free surface" problems and gives the possibility to solve real two-fluid problems that a free surface formulation cannot represents.

For the case of two immiscible fluids, the discontinuity in the material properties when the interface cuts an element leads to discontinuities in the gradients of the unknowns that standard interpolations cannot capture. A local pressure enriched function was tested to solve the interaction of viscoelastic and Newtonian fluids with very good results, correcting the amount of lost mass, and permitting the exact resolution of the hydrostatic rest state that standard formulations cannot solve.

The ten degrees of freedom per node in a three-dimensional case of the viscoelastic fluid flow problem requires efficient algorithms to resolve the coupled system of equations. In this work a fractional step approach to solve the two immiscible fluid flow problem was tested which shows an important reduction in computational resources with respect to the monolithic approach, both in the total CPU time required to solve the problem and in the total memory necessary to store the matrices.

A discontinuity-capturing technique for the constitutive equation was tested in order to eliminate nonphysical peaks that can appear when the flow finds an abrupt change in the geometry with very satisfactory results.

\section{Acknoledgments}

E. Castillo gratefully acknowledges the support received from CONICYT (Programa de Formaciï ¿œn de Capital Humano Avanzado/Becas-Chile Folio 72120080), and from the Universidad de Santiago de Chile. R. Codina gratefully acknowledges the support received from the Catalan Goverment through the ICREA 
Acadèmia Research Program. This work was partially funded by the by the Ministerio de Economï̈œa $y$ Competitividad of the Spanish Government under the Plan Nacional de Investigaciï ¿œn 2012: AYA201233490 .

\section{References}

[1] S. Osher, R. Fedkiwr, in: Level Set Methods and Dynamic Implicit Surfaces, Springer-Verlag, 2003.

[2] M. Cruchaga, L. Battaglia, M. Storti, J. D'Elía, Numerical modeling and experimental validation of free surface flow problems, Archives of Computational Methods in Engineering (2014) 1-31.

[3] J.-D. Yu, S. Sakai, J. Sethian, Two-phase viscoelastic jetting, Journal of Computational Physics 220 (2) (2007) 568-585.

[4] S. Pillapakkam, P. Singh, A Level-Set Method for Computing Solutions to Viscoelastic Two-Phase Flow, Journal of Computational Physics 174 (2) (2001) 552-578.

[5] A. Bonito, M. Picasso, M. Laso, Numerical simulation of 3D viscoelastic flows with free surfaces, Journal of Computational Physics 215 (2) (2006) 691-716.

[6] W. Noh, P. Woodward, SLIC (Simple Line Interface Calculation), Vol. 59 of Lecture Notes in Physics, Springer Berlin Heidelberg, 1976, pp. 330-340.

[7] M. F. Tomé, S. McKee, GENSMAC: A Computational Marker and Cell Method for Free Surface Flows in General Domains, Journal of Computational Physics 110 (1) (1994) 171-186.

[8] M. Tomé, A. Filho, J. Cuminato, N. Mangiavacchi, S. McKee, GENSMAC3D: a numerical method for solving unsteady three-dimensional free surface flows, International Journal for Numerical Methods in Fluids 37 (7) (2001) 747-796.

[9] M. Tomé, A. Castelo, A. Afonso, M. Alves, F. Pinho, Application of the log-conformation tensor to threedimensional time-dependent free surface flows, Journal of Non-Newtonian Fluid Mechanics 175-176 (0) (2012) 44-54.

[10] R. Figueiredo, C. Oishi, J. Cuminato, M. Alves, Three-dimensional transient complex free surface flows: Numerical simulation of XPP fluid, Journal of Non-Newtonian Fluid Mechanics 195 (0) (2013) 88-98.

[11] S. McKee, M. Tomé, V. Ferreira, J. Cuminato, A. Castelo, F. Sousa, N. Mangiavacchi, The MAC method, Computers \& Fluids 37 (8) (2008) 907 - 930. doi:http://dx.doi.org/10.1016/j.compfluid.2007.10.006.

URL http://www.sciencedirect.com/science/article/pii/S0045793007001909

[12] P. Minev, T. Chen, K. Nandakumar, A finite element technique for multifluid incompressible flow using Eulerian grids, Journal of Computational Physics 187 (1) (2003) 255-273.

[13] J. Chessa, T. Belytschko, An Extended Finite Element Method for Two-Phase Fluids, Journal of Applied Mechanics 70 (2003) 10-17. 
[14] A. H. Coppola-Owen, R. Codina, Improving Eulerian two-phase flow finite element approximation with discontinuous gradient pressure shape functions, International Journal for Numerical Methods in Fluids 49 (12) (2005) 1287-1304.

[15] M. Sussman, A second order coupled level set and volume-of-fluid method for computing growth and collapse of vapor bubbles, Journal of Computational Physics 187 (1) (2003) 110-136.

[16] S. P. van der Pijl, A. Segal, C. Vuik, P. Wesseling, A mass-conserving Level-Set method for modelling of multi-phase flows, International Journal for Numerical Methods in Fluids 47 (4) (2005) 339-361.

[17] E. Olsson, G. Kreiss, A conservative level set method for two phase flow, Journal of Computational Physics 210 (1) (2005) 225-246.

[18] R. Codina, O. Soto, A numerical model to track two-fluid interfaces based on a stabilized finite element method and the level set technique, International Journal for Numerical Methods in Fluids 4 (2002) 293-301.

[19] M. Sussman, E. G. Puckett, A Coupled Level Set and Volume-of-Fluid Method for Computing 3D and Axisymmetric Incompressible Two-Phase Flows, Journal of Computational Physics 162 (2) (2000) $301-337$.

[20] R. Codina, Finite element approximation of the three-field formulation of the Stokes problem using arbitrary interpolations, SIAM Journal on Numerical Analysis 47 (1) (2009) 699-718.

[21] E. Castillo, R. Codina, Stabilized stress-velocity-pressure finite element formulations of the Navier-Stokes problem for fluids with non-linear viscosity, Computer Methods in Applied Mechanics and Engineering 279 (0) (2014) 554-578.

[22] J. Marchal, M. Crochet, A new mixed finite element for calculating viscoelastic flow, Journal of NonNewtonian Fluid Mechanics 26 (1) (1987) 77-114.

[23] M. Fortin, A. Fortin, A new approach for the FEM simulation of viscoelastic flows, Journal of NonNewtonian Fluid Mechanics 32 (3) (1989) 295-310.

[24] Y. Fan, R. Tanner, N. Phan-Thien, Galerkin/least-square finite-element methods for steady viscoelastic flows, Journal of Non-Newtonian Fluid Mechanics 84 (2-3) (1999) 233-256.

[25] J. Y. Yoo, Y. Na, A numerical study of the planar contraction flow of a viscoelastic fluid using the SIMPLER algorithm, Journal of Non-Newtonian Fluid Mechanics 39 (1) (1991) 89-106.

[26] M. Alves, F. Pinho, P. Oliveira, Effect of a high-resolution differencing scheme on finite-volume predictions of viscoelastic flows, Journal of Non-Newtonian Fluid Mechanics 93 (2-3) (2000) 287-314.

[27] E. Castillo, R. Codina, Variational multi-scale stabilized formulations for the stationary three-field incompressible viscoelastic flow problem, Computer Methods in Applied Mechanics and Engineering 279 (0) (2014) 579-605.

[28] R. Fattal, R. Kupferman, Constitutive laws for the matrix-logarithm of the conformation tensor, Journal of Non-Newtonian Fluid Mechanics 123 (2-3) (2004) 281-285. 
[29] M. Tomé, A. Castelo, V. Ferreira, S. McKee, A finite difference technique for solving the Oldroyd-B model for 3d-unsteady free surface flows, Journal of Non-Newtonian Fluid Mechanics 154 (2-3) (2008) 179-206.

[30] F. Habla, M. W. Tan, J. Haßlberger, O. Hinrichsen, Numerical simulation of the viscoelastic flow in a three-dimensional lid-driven cavity using the log-conformation reformulation in OpenFOAM, Journal of Non-Newtonian Fluid Mechanics 212 (0) (2014) 47-62.

[31] J. S. Howell, Computation of viscoelastic fluid flows using continuation methods, Journal of Computational and Applied Mathematics 225 (1) (2009) 187-201.

[32] E. Carew, P. Townsend, M. Webster, On a discontinuity capturing technique for Oldroyd-B fluids, Journal of Non-Newtonian Fluid Mechanics 51 (2) (1994) 231-238.

[33] R. B. Bird, R. C. Amstrong, O. Hassager, in: Dynamics of Polymeric Liquids, vol.1: Fluid Mechanics, 2nd Edition, Wiley, New York, 1987.

[34] R. B. Bird, C. F. Curtiss, R. C. Amstrong, O. Hassager, in: Dynamics of Polymeric Liquids, vol.2: Kinetic Theory, 2nd Edition, Wiley, New York, 1987.

[35] Y. Mu, G. Zhao, X. Wu, J. Zhai, Modeling and simulation of three-dimensional planar contraction flow of viscoelastic fluids with PTT, Giesekus and FENE-P constitutive models, Applied Mathematics and Computation 218 (17) (2012) 8429-8443.

[36] E. Fernández-Cara, F. Guillén, R. Ortega, Mathematical modeling and analysis of viscoelastic fluids of the Oldroyd kind, in Handbook of Numerical Analysis, VIII, North-Holland, 2002.

[37] J. Bonvin, M. Picasso, R. Stenberg, GLS and EVSS methods for a three-field Stokes problem arising from viscoelastic flows, Computer Methods in Applied Mechanics and Engineering 190 (29-30) (2001) 3893-3914.

[38] E. Castillo, R. Codina, First, second and third order fractional step methods for the three-field viscoelastic flow problem, Journal of Computational Physics 296 (0) (2015) 113 - 137.

[39] R. Codina, Analysis of a stabilized finite element approximation of the Oseen equations using orthogonal subscales, Applied Numerical Mathematics 58 (2008) 264-283.

[40] R. Guénette, M. Fortin, A new mixed finite element method for computing viscoelastic flows, Journal of Non-Newtonian Fluid Mechanics 60 (1995) 27-52.

[41] S. Badia, R. Codina, Algebraic pressure segregation methods for the incompressible Navier-Stokes equations, Archives of Computational Methods in Engineering 15 (2008) 343-369.

[42] A. N. Brooks, T. J. Hughes, Streamline Upwind/Petrov-Galerkin formulations for convection dominated flows with particular emphasis on the incompressible Navier-Stokes equations, Computer Methods in Applied Mechanics and Engineering 32 (1-3) (1982) 199-259.

[43] H. A. van der Vorst, BI-CGSTAB: A Fast and Smoothly Converging Variant of BI-CG for the solution of Nonsymmetric Linear Systems, SIAM J. Sci. Stat. Comput. 13 (2) (1992) 631-644. 
[44] M. Cruchaga, D. Celentano, T. Tezduyar, Collapse of a liquid column: Numerical simulation and experimental validation, Computational Mechanics 39 (4) (2007) 453-476.

[45] N. O. Moraga, L. A. Lemus, M. A. Saavedra, R. A. Lemus-Mondaca, VOF/FVM prediction and experimental validation for shear-thinning fluid column collapse, Computers \& Mathematics with Applications 69 (2) (2015) 89-100.

[46] M. F. Tomé, S. McKee, Numerical simulation of viscous flow: Buckling of planar jets, International Journal for Numerical Methods in Fluids 29 (6) (1999) 705-718.

[47] J. O. Cruickshank, B. R. Munson, Viscous fluid buckling of plane and axisymmetric jets, Journal of Fluid Mechanics 113 (1981) 221-239. 